\title{
FREE OPERATED MONOIDS AND REWRITING SYSTEMS
}

\author{
JIN ZHANG AND XING GAO *
}

\begin{abstract}
Aвstract. The construction of bases for quotients is an important problem. In this paper, applying the method of rewriting systems, we give a unified approach to construct sections-an alternative name for bases in semigroup theory - for quotients of free operated monoids. As applications, we capture sections of free $*$-monoids and free groups, respectively.
\end{abstract}

\section{Contents}

1. Introduction

2. Rewrting systems and sections

2.1. Operated monoids and operated congruences

2.2. Relationship between rewriting systems and sections

3. Applications

3.1. $\quad$ Free $*$-monoids

3.2. Free groups

References

\section{InTRODUCTION}

In 1960, A. G. Kurosh [23] first introduced the concept of algebras with one or more linear operators. An example of such algebras is the differential algebra led by the algebraic abstraction of differential operator in analysis. Differential algebra is from a purely algebraic viewpoint to study differentiation and nonlinear differential equations without using an underlying topology, and has been largely successful in many crucial areas, such as uncoupling of nonlinear systems, classification of singular components, and detection of hidden equations [22, 28]. Another important example of such algebras is the Rota-Baxter algebra (first called Baxter algebra) which is the algebraic abstraction of integral operator in analysis [17]. Rota-Baxter algebra, originated from probability study [5], is related beautifully to the classical Yang-Baxter equation, as well as to operads, to combinatorics and, through the Hopf algebra framework of Connes and Kreimer, to the renormalization of quantum field theory [1, 3, 4, 11, 12, 19]. Other examples are also important, such as averaging algebra, Reynolds algebras, Nijenhuis algebras and Leroux's TD algebras [8, 26, 25]. Each of the above examples is an algebra with one linear operator, which is named operated algebras by Guo [16].

Definition 1.1. An operated monoid (resp. operated k-algebra) is a monoid (resp. k-algebra) $U$ together with a map (resp. k-linear map) $P_{U}: U \rightarrow U$, where $\mathbf{k}$ is a commutative unitary ring.

In that paper [16], Guo constructed the free operated $\mathbf{k}$-algebra on a set. Since the free operated $\mathbf{k}$-algebra as modules is precisely the free k-module with basis the free operated monoid, the crucial step of Guo's method is to construct the free operated monoid on a set-the main object considered in this paper, See also [7, 13].

Date: November 9, 2018.

2010 Mathematics Subject Classification. 16S15, 08A70, 06F05, $20 \mathrm{M} 05$.

Key words and phrases. Operated monoids, term-rewriting systems, free $*$-monoids, free groups.

*Corresponding author. 
Abstract rewriting system is a branch of theoretical computer science, combining elements of logic, universal algebra, automated theorem proving and functional programming [2, 27]. The theory of convergent rewriting systems is successfully applied to find bases of free differential type [18] and free Rota-Baxter type algebras [14], which reveals the power of rewriting systems in the study of operators.

Let us point out that groups can be viewed as operated monoids if one considers the inverse operator as a map from the group to itself. In the same way, many other important classes of monoids such as inverse monoids, I-monoids and $*$-monoids can also be fitted into the framework of operated monoids. All of these examples can be obtained from free operated monoids by taking quotients modulo suitable operated congruences. It is interesting to find bases for quotients. The bases of quotients in semigroup theory are also called sections. In the present paper we obtained a method, in terms of convergent rewriting systems, to give sections for quotients of free operated monoids. Our method is parallel to the famous Composition-Diamond lemma in Gröbner-Shirshov theory [7]. As applications, we capture sections of free $*$-monoids and free groups, respectively.

The organisation of this paper is as follows. In Section 2 , after reviewing the construction of free operated monoids, we characterize the operated congruence generated by a binary relation on a free operated monoid (Proposition 2.10). Then we associate to each binary relation on a free operated monoid a rewriting system (Definition 2.14). We also establish a relationship between convergent rewriting systems on free operated monoids and sections of quotients of free operated monoids. (Theorem 2.21). In Section 3, as applications of our main result, we acquire respectively sections of free $*$-monoids (Theorem 3.10 ) and free groups (Theorem 3.15).

\section{REWRTING SYSTEMS AND SECTIONS}

In this section, based on rewriting systems on free operated monoids $\mathfrak{M}(X)$, we give an approach to construct sections for quotients of $\mathfrak{M}(X)$.

2.1. Operated monoids and operated congruences. The construction of free operated monoids was given in [16, 18]. See also [7]. We reproduce that construction here to review the notations. For any set $Y$, denote by $M(Y)$ the free monoid on $Y$.

Let $X$ be a set. We proceed via finite stages $\mathfrak{M}_{n}(X)$ defined recursively to construct the free operated monoid $\mathfrak{M}(X)$ on $X$. The initial stage is $\mathfrak{M}_{0}(X):=M(X)$ and $\mathfrak{M}_{1}(X):=M\left(X \cup\left\lfloor\mathfrak{M}_{0}(X)\right\rfloor\right)$, where $\left\lfloor\mathfrak{M}_{0}(X)\right\rfloor:=\left\{\lfloor u\rfloor \mid u \in \mathfrak{M}_{0}(X)\right\}$ is a disjoint copy of $\mathfrak{M}_{0}(X)$. The inclusion $X \hookrightarrow X \cup\left\lfloor\mathfrak{M}_{0}\right\rfloor$ induces a monomorphism

$$
i_{0}: \mathfrak{M}_{0}(X)=M(X) \hookrightarrow \mathfrak{M}_{1}(X)=M\left(X \cup\left\lfloor\mathfrak{M}_{0}\right\rfloor\right)
$$

of monoids through which we identify $\mathfrak{M}_{0}(X)$ with its image in $\mathfrak{M}_{1}(X)$.

For $n \geqslant 1$, assume inductively that $\mathfrak{M}_{n}(X)$ has been defined and the embedding

$$
i_{n-1, n}: \mathfrak{M}_{n-1}(X) \hookrightarrow \mathfrak{M}_{n}(X)
$$

has been obtained. Then we define

$$
\mathfrak{M}_{n+1}(X):=M\left(X \cup\left\lfloor\mathfrak{M}_{n}(X)\right\rfloor\right) .
$$

Since $\mathfrak{M}_{n}(X)=M\left(X \cup\left\lfloor\mathfrak{M}_{n-1}(X)\right\rfloor\right)$ is a free monoid, the injection

$$
\left\lfloor\mathfrak{M}_{n-1}(X)\right\rfloor \hookrightarrow\left\lfloor\mathfrak{M}_{n}(X)\right\rfloor
$$


induces a monoid embedding

$$
\mathfrak{M}_{n}(X)=M\left(X \cup\left\lfloor\mathfrak{M}_{n-1}(X)\right\rfloor\right) \hookrightarrow \mathfrak{M}_{n+1}(X)=M\left(X \cup\left\lfloor\mathfrak{M}_{n}(X)\right\rfloor\right) .
$$

Finally we define the monoid

$$
\mathfrak{M}(X):=\lim _{\longrightarrow} \mathfrak{M}_{n}=\bigcup_{n \geqslant 0} \mathfrak{M}_{n}(X),
$$

whose elements are called bracketed words on $X$. Elements $w \in \mathfrak{M}_{n} \backslash \mathfrak{M}_{n-1}$ are said to have depth $n$, denoted by $\operatorname{dep}(w)=n$.

Lemma 2.1. ([17]) Every bracketed word $w \neq 1$ has a unique decomposition $w=w_{1} \cdots w_{m}$, where $w_{i}, 1 \leqslant i \leqslant m$, is in $X$ or in $\lfloor\mathfrak{M}(X)\rfloor:=\{\lfloor w\rfloor \mid w \in \mathfrak{M}(X)\}$. We call $|w|:=m$ the breadth of $w$.

The following result shows that $\mathfrak{M}(X)$ is the free object in the category of operated monoids.

Lemma 2.2. ([16, 17]) Let $i_{X}: X \rightarrow \mathfrak{M}(X)$ be the natural embeddings. Then the triple $\left(\mathfrak{M}(X),\llcorner\rfloor, i_{X}\right)$ is the free operated monoid on $X$, where

$$
\lfloor\rfloor: \mathfrak{M}(X) \rightarrow \mathfrak{M}(X), w \mapsto\lfloor w\rfloor
$$

is an operator on $\mathfrak{M}(X)$.

The concept of $\star$-bracketed words plays a crucial role in the theory of Gröbner-Shirshov bases [6].

Definition 2.3. Let $X$ be a set, $\star$ a symbol not in $X$ and $X^{\star}=X \cup\{\star\}$.

(a) By a $\star$-bracketed word on $X$, we mean any bracketed word in $\mathfrak{M}\left(X^{\star}\right)$ with exactly one occurrence of $\star$, counting multiplicities. The set of all $\star$-bracketed words on $X$ is denoted by $\mathfrak{M}^{\star}(X)$.

(b) For $q \in \mathfrak{M}^{\star}(X)$ and $u \in \mathfrak{M}(X)$, we define $\left.q\right|_{\star \mapsto u}$ to be the bracketed word on $X$ obtained by replacing the symbol $\star$ in $q$ by $u$, for convenience, denoted it by $\left.q\right|_{u}$.

(c) Let $u, v \in \mathfrak{M}(X)$. We say that $u$ is a bracketed subword of $v$, if there exist $q \in \mathfrak{M}^{\star}(X)$ such that $v=\left.q\right|_{u}$.

Generally, with $\star_{1}, \star_{2}$ distinct symbols not in $X$, set $X^{\star 2}:=X \cup\left\{\star_{1}, \star_{2}\right\}$.

(d) We define an $\left(\star_{1}, \star_{2}\right)$-bracketed word on $X$ to be a bracketed word in $\mathfrak{M}\left(X^{\star 2}\right)$ with exactly one occurrence of each of $\star_{i}, i=1,2$. The set of all $\left(\star_{1}, \star_{2}\right)$-bracketed words on $X$ is denoted by $\mathfrak{M}^{\star_{1}, \star_{2}}(X)$.

(e) For $q \in \mathfrak{M}^{\star_{1}, \star_{2}}(X)$ and $u_{1}, u_{2} \in \mathfrak{M}^{\star_{1}, \star_{2}}(X)$, we define

$$
\left.q\right|_{u_{1}, u_{2}}:=\left.q\right|_{\star_{1} \mapsto u_{1}, \star_{2} \mapsto u_{2}}
$$

to be obtained by replacing the letters $\star_{i}$ in $q$ by $u_{i}$ for $i=1,2$.

Remark 2.4. If $\left.p\right|_{u}=\left.p\right|_{v}$ with $p \in \mathfrak{M}^{\star}(X)$ and $u, v \in \mathfrak{M}(X)$, then $u=v$ by the freeness of $\mathfrak{M}(X)$.

The concept of operated congruences will be used throughout the paper.

Definition 2.5. An equivalence $R$ on $\mathfrak{M}(X)$ is operated congruence if

(C1) $(\forall a, b, c \in \mathfrak{M}(X))(a, b) \in R \Rightarrow(a c, b c) \in R$;

(C2) $(\forall a, b, c \in \mathfrak{M}(X))(a, b) \in R \Rightarrow(c a, c b) \in R$;

(C3) $(\forall a, b \in \mathfrak{M}(X))(a, b) \in R \Rightarrow(\lfloor a\rfloor,\lfloor b\rfloor) \in R$. 
Let $R$ be a binary relation on $\mathfrak{M}(X)$. There is a unique smallest operated congruence $\langle R\rangle$ on $\mathfrak{M}(X)$ containing $R$, which will be described in the following. Define

$$
R^{c}:=\left\{\left(\left.q\right|_{a},\left.q\right|_{b}\right) \mid q \in \mathfrak{M}^{\star}(X),(a, b) \in R\right\} .
$$

We record some basic properties of $R^{c}$. For any $u \in \mathfrak{M}(X)$, define recursively $\lfloor u\rfloor^{(0)}:=u$ and $\lfloor u\rfloor^{(k+1)}:=\left\lfloor\lfloor u\rfloor^{(k)}\right\rfloor$ for $k \geqslant 0$.

Lemma 2.6. $R^{c}$ is the smallest binary relation containing $R$ and satisfy $(C 1),(C 2)$ and $(C 3)$.

Proof. According to the definition of $R^{c}$, we have $R \subseteq R^{c}$ by choosing $q=\star$. Let $c \in \mathfrak{M}(X)$ and

$$
\left(\left.q\right|_{a},\left.q\right|_{b}\right) \in R^{c} \text { with } q \in \mathfrak{M}^{\star}(X),(a, b) \in R .
$$

Write $q_{1}:=q c$. Then by Eq. (1),

$$
\left(\left(\left.q\right|_{a}\right) c,\left(\left.q\right|_{b}\right) c\right)=\left(\left.(q c)\right|_{a},\left.(q c)\right|_{b}\right)=\left(\left.q_{1}\right|_{a},\left.q_{1}\right|_{b}\right) \in R^{c} .
$$

So $R^{c}$ satisfies $(\mathrm{C} 1)$. By symmetry, $R^{c}$ also satisfies $(\mathrm{C} 2)$. To prove $R^{c}$ satisfies $(\mathrm{C} 3)$, let $q_{2}:=$ $\lfloor q\rfloor \in \mathfrak{M}^{\star}(X)$. Then again from Eq. (1),

$$
\left(\left\lfloor\left. q\right|_{a}\right\rfloor,\left\lfloor\left. q\right|_{b}\right\rfloor\right)=\left(\left.q_{2}\right|_{a},\left.q_{2}\right|_{b}\right) \in R^{c} .
$$

Suppose that $T$ is a binary relation containing $R$ and satisfying (C1), (C2) and (C3). Let $\left(\left.q\right|_{a},\left.q\right|_{b}\right) \in R^{c}$ with $q \in \mathfrak{M}^{\star}(X)$ and $(a, b) \in R$. Then $(a, b) \in T$ by $R \subseteq T$. Hence $\left(\left.q\right|_{a},\left.q\right|_{b}\right) \in T$ by $(\mathrm{C} 1),(\mathrm{C} 2)$ and (C3).

For a binary relation $R$ on $\mathfrak{M}(X)$, write $R^{-1}:=\{(b, a) \mid(a, b) \in R\}$.

Lemma 2.7. Let $R, S$ be binary relations on $\mathfrak{M}(X)$. Then

(a) $R \subseteq S \Rightarrow R^{c} \subseteq S^{c}$;

(b) $\left(R^{-1}\right)^{c}=\left(R^{c}\right)^{-1}$;

(c) $(R \cup S)^{c}=R^{c} \cup S^{c}$.

Proof. (稀) This follows directly from Eq. (1).

(b) Let $\left(\left.q\right|_{a},\left.q\right|_{b}\right) \in\left(R^{-1}\right)^{c}$ with $(a, b) \in R^{-1}$ and $q \in \mathfrak{M}^{\star}(X)$. Then

$$
(b, a) \in R,\left(\left.q\right|_{b},\left.q\right|_{a}\right) \in R^{c} \text { and }\left(\left.q\right|_{a},\left.q\right|_{b}\right) \in\left(R^{c}\right)^{-1},
$$

and so $\left(R^{-1}\right)^{c} \subseteq\left(R^{c}\right)^{-1}$. Conversely, let $\left(\left.q\right|_{a},\left.q\right|_{b}\right) \in\left(R^{c}\right)^{-1}$ with $q \in \mathfrak{M}^{\star}(X)$. Then

$$
\left(\left.q\right|_{b},\left.q\right|_{a}\right) \in R^{c},(b, a) \in R \text { and }(a, b) \in R^{-1},
$$

which implies that

$$
\left(\left.q\right|_{a},\left.q\right|_{b}\right) \in\left(R^{-1}\right)^{c} \text { and }\left(R^{c}\right)^{-1} \subseteq\left(R^{-1}\right)^{c},
$$

as needed.

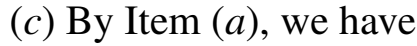

$$
R^{c} \subseteq(R \cup S)^{c} \text { and } S^{c} \subseteq(R \cup S)^{c},
$$

and thus $R^{c} \cup S^{c} \subseteq(R \cup S)^{c}$. Conversely, let $\left(\left.q\right|_{a},\left.q\right|_{b}\right) \in(R \cup S)^{c}$ with $(a, b) \in R \cup S$. Then

$$
(a, b) \in R \text { or }(a, b) \in S \text { and so }\left(\left.q\right|_{a},\left.q\right|_{b}\right) \in R^{c} \text { or }\left(\left.q\right|_{a},\left.q\right|_{b}\right) \in S^{c} \text {. }
$$

Hence $\left(\left.q\right|_{a},\left.q\right|_{b}\right) \in R^{c} \cup S^{c}$ and $(R \cup S)^{c} \subseteq R^{c} \cup S^{c}$.

Lemma 2.8. Let $R$ be a binary relation on $\mathfrak{M}(X)$ satisfying $(C 1),(C 2)$ and $(C 3)$. Then, so is $R^{n}(=R \circ R \circ \cdots \circ R)$ for all $n \geqslant 1$. 
Proof. Let $(a, b) \in R^{n}$. Then there exist $v_{1}, v_{2}, \cdots, v_{n-1}$ in $\mathfrak{M}(X)$ such that

$$
\left(a, v_{1}\right),\left(v_{1}, v_{2}\right), \cdots,\left(v_{n-1}, b\right) \in R .
$$

Because $R$ satisfies (C1),(C2) and (C3), it follows that, for all $c$ in $\mathfrak{M}(X)$,

$$
\begin{gathered}
\left(c a, c v_{1}\right),\left(c v_{1}, c v_{2}\right), \cdots,\left(c v_{n-1}, c b\right) \in R, \\
\left(a c, v_{1} c\right),\left(v_{1} c, v_{2} c\right), \cdots,\left(v_{n-1} c, b c\right) \in R, \\
\left(\lfloor a\rfloor,\left\lfloor v_{1}\right\rfloor\right),\left(\left\lfloor v_{1}\right\rfloor,\left\lfloor v_{2}\right\rfloor\right), \cdots,\left(\left\lfloor v_{n-1}\right\rfloor,\lfloor b\rfloor\right) \in R .
\end{gathered}
$$

So

as required.

$$
(c a, c b),(a c, a b),(\lfloor a\rfloor,\lfloor b\rfloor) \in R^{n},
$$

We recall the construction of equivalences $R^{e}$ generated by a binary relation $R$ on a set $X[21]$. Write

$$
R^{\infty}:=\cup_{n \geqslant 1} R^{n} \text { and } 1_{X}:=\{(x, x) \mid x \in X\} .
$$

Lemma 2.9. ([21] ) Let $R$ be a binary relation on a set $X$. Then $R^{e}=\left[R \cup R^{-1} \cup 1_{X}\right]^{\infty}$. More precisely, $(x, y) \in R^{e}$ if and only if either $x=y$ or, for some $n$ in $\mathbf{N}$, there is a sequence of elements

$$
x=z_{1}, z_{2}, \cdots, z_{n}=y,
$$

in which, for each $i$ in $\{1,2, \cdots, n-1\}$, either $\left(z_{i}, z_{i+1}\right) \in R$ or $\left(z_{i+1}, z_{i}\right) \in R$.

Now we arrive at the position to give the description of operated congruences generated by binary relations.

Proposition 2.10. For every binary relation $R$ on $\mathfrak{M}(X),\langle R\rangle=\left(R^{c}\right)^{e}$.

Proof. By Lemma 2.9, $\left(R^{c}\right)^{e}$ is an equivalence containing $R^{c}$, and so certainly containing $R$ by Lemma 2.6. Next we show that $\left(R^{c}\right)^{e}$ satisfies $(\mathrm{C} 1),(\mathrm{C} 2)$ and $(\mathrm{C} 3)$. From Lemma 2.9,

$$
\left(R^{c}\right)^{e}=S^{\infty}=\cup_{n \geqslant 1} S^{n}, \text { where } S=R^{c} \cup\left(R^{c}\right)^{-1} \cup 1_{\mathfrak{M}(X)} .
$$

Using Lemma2.7 and the fact that $1_{\mathfrak{M}(X)}=1_{\mathfrak{M}(X)}^{c}$, we get

$$
S=R^{c} \cup\left(R^{c}\right)^{-1} \cup 1_{\mathfrak{M}(X)}=R^{c} \cup\left(R^{-1}\right)^{c} \cup 1_{\mathfrak{M}(X)}^{c}=\left(R \cup R^{-1} \cup 1_{\mathfrak{M}(X)}\right)^{c},
$$

which implies from Lemma 2.6 that $S$ satisfies (C1), (C2) and (C3), and so is $S^{n}$ for all $n \geqslant 1$ by Lemma 2.8. Hence

$$
\left(R^{c}\right)^{e}=S^{\infty}=\cup_{n \geqslant 1} S^{n}
$$

also satisfies $(\mathrm{C} 1),(\mathrm{C} 2)$ and $(\mathrm{C} 3)$. Moreover since $\left(R^{c}\right)^{e}$ is already an equivalence, it is an operated congruence by Definition 2.5 .

Finally, suppose $T$ is an arbitrary operated congruence on $\mathfrak{M}(X)$ containing $R$. Then $T^{c}=T$ by Definition 2.5 and Eq. (1). So from Lemma 2.7.

$$
R^{c} \subseteq T^{c}=T \text { and }\left(R^{c}\right)^{e} \subseteq T^{e}=T .
$$

This completes the proof.

Proposition 2.11. Let $R$ be a binary relation on $\mathfrak{M}(X)$. Then $(a, b) \in\langle R\rangle$ if and only if either $a=b$ or, for some $n$ in $\mathbf{N}$, there is a sequence of elements $a=v_{1}, v_{2}, \cdots, v_{n}=b$, in which, for each $i$ in $\{1,2, \cdots, n-1\}$, either $\left(v_{i}, v_{i+1}\right) \in R^{c}$ or $\left(v_{i+1}, v_{i}\right) \in R^{c}$.

Proof. It follows from Lemmas 2.9 and 2.10. 
Every operated congruence $R$ on $\mathfrak{M}(X)$ has a corresponding quotient structure $\mathfrak{M}(X) / R$, whose elements are operated congruence classes for the relation. We end this subsection with an important concepts used later.

Definition 2.12. Let $X$ be a set and $R$ an operated congruence on $\mathfrak{M}(X)$. We call $W \subseteq \mathfrak{M}(X)$ a section of $R$ if, for each operated congruence class $A$ of $\mathfrak{M}(X) / R$, there exists exactly one element $w \in W$ such that $w \in A$.

2.2. Relationship between rewriting systems and sections. In this subsection, we first associate a rewriting system $\Pi_{S}$ to a binary relation $S$ on $\mathfrak{M}(X)$. A monomial order compatible with all operations is needed.

Definition 2.13. Let $X$ be a set. A monomial order on $\mathfrak{M}(X)$ is a well-ordering $\leqslant$ on $\mathfrak{M}(X)$ such that

$$
u<v \Longrightarrow u w<v w, w u<w v,\lfloor u\rfloor<\lfloor v\rfloor \text { for all } u, v, w \in \mathfrak{M}(X) .
$$

We denote $u<v$ if $u \leqslant v$ but $u \neq v$.

The following concepts are adapted from [2, 14].

Definition 2.14. Let $X$ be a set and $\leqslant$ a monomial order on $\mathfrak{M}(X)$. Let $S$ be a binary relation on $\mathfrak{M}(X)$.

(a) A term-rewriting system $\Pi_{S}$ on $\mathfrak{M}(X)$ associated to $S$ is a binary relation of $\mathfrak{M}(X)$, denote by

$$
\Pi_{S}:=\left\{\left(\left.q\right|_{t},\left.q\right|_{v}\right) \mid q \in \mathfrak{M}^{\star}(X),(t, v) \in S \cup S^{-1}, t>v\right\} .
$$

An element in $\Pi_{S}$ is called a rewriting rule .

(b) Let $f, g \in \mathfrak{M}(X)$, we call $f$ rewrites to $g$ in one-step with respect to $\Pi_{S}$, if $(f, g) \in \Pi_{S}$. We indicate any such one-step rewriting by $f \rightarrow_{\Pi_{S}} g$.

(c) The reflexive transitive closure of $\Pi_{S}$ (as a binary relation on $\mathfrak{M}(X)$ ) will be denoted by $\stackrel{*}{\rightarrow} \Pi_{S}$ and we say $f$ rewrites to $g$ with respect to $\Pi_{S}$ if $f \stackrel{*}{\rightarrow}_{\Pi_{S}} g$. In this case, we call $f$ is a predecessor of $g$. Denote by $\mathcal{P}(g)$ the set of all predecessors of $g$. Note that $g \in \mathcal{P}(g)$.

(d) Two elements $f, g \in \mathfrak{M}(X)$ are joinable if there exists $h \in \mathfrak{M}(X)$ such that $f \stackrel{*}{\rightarrow} \Pi_{S} h$ and $g \stackrel{*}{\rightarrow} \Pi_{S} h$. Denote it by $f \downarrow_{\Pi_{S}} g$.

(e) The image $\pi_{1}\left(\Pi_{S}\right)$ under the first projection map will be denoted by $\operatorname{Dom}\left(\Pi_{S}\right)$. An element $f \in \mathfrak{M}(X)$ is irreducible or in normal form if $f \notin \operatorname{Dom}\left(\Pi_{S}\right)$, that is, no more rewriting rule from $\Pi_{S}$ can apply to $f$.

Definition 2.15. The term-rewriting system $\Pi_{S}$ defined above is called

(a) terminating if there is no infinite chain of one-setp rewriting

$$
f_{0} \rightarrow_{\Pi_{S}} f_{1} \rightarrow_{\Pi_{S}} f_{2} \cdots \text {; }
$$

(b) confluent if every fork $\left(f \stackrel{*}{\rightarrow} \Pi_{S} h, f \stackrel{*}{\rightarrow}_{\Pi_{S}} g\right)$, we have $g \downarrow_{\Pi_{S}} h$;

(c) locally confluent if for every local fork $\left(f \rightarrow_{\Pi_{S}} h, f \rightarrow_{\Pi_{S}} g\right)$, we have $g \downarrow_{\Pi_{S}} h$;

(d) convergent if it is both terminating and confluent.

A well-known result on rewriting systems is Newman's Lemma [2, Lemma 2.7.2].

Lemma 2.16. (Newman) A terminating rewriting system is confluent if and only if it is locally confluent. 
Lemma 2.17. Let $X$ be a set and $\leqslant$ a monomial order on $\mathfrak{M}(X)$. Let $S$ be a binary relation on $\mathfrak{M}(X)$. The term-rewriting system $\Pi_{S}$ defined in Eq. (3) is terminating.

Proof. Suppose to the contrary that $\Pi_{S}$ is not terminating. Then there is an infinite chain of one-step rewritings

$$
f_{0} \rightarrow_{\Pi_{S}} f_{1} \rightarrow_{\Pi_{S}} f_{2} \cdots
$$

Let $f \rightarrow_{\Pi_{S}} g$ be a one-step rewriting. Then $(f, g) \in \Pi_{S}$ and so we can write $(f, g)=\left(\left.q\right|_{t},\left.q\right|_{v}\right)$ with $q \in \mathfrak{M}^{\star}(X),(t, v) \in S \cup S^{-1}$ and $t>v$. Since $\leqslant$ is a monomial order, we have $f=\left.q\right|_{t}>\left.q\right|_{v}=g$. Hence

$$
f_{0}>f_{1}>f_{2} \cdots,
$$

contradicting that $\leqslant$ is a well-order.

We are going to capture the relationship between convergent term-rewriting systems on $\mathfrak{M}(X)$ and sections of quotients of $\mathfrak{M}(X)$. Let us record three lemmas as a preparation.

Lemma 2.18. Let $X$ be a set and $\leqslant$ a monomial order on $\mathfrak{M}(X)$. Let $S$ be a binary relation on $\mathfrak{M}(X)$. If $a \stackrel{*}{\rightarrow} \Pi_{S} b$ with $a, b \in \mathfrak{M}(X)$, then $(a, b) \in\langle S\rangle$.

Proof. If $a=b$, then $(a, b) \in\langle S\rangle$. Suppose $a \neq b$. Then there are $a_{1}, a_{2}, \cdots, a_{n} \in \mathfrak{M}(X)$ such that

$$
a=a_{1} \rightarrow_{\Pi_{S}} a_{2} \rightarrow_{\Pi_{S}} \cdots \rightarrow_{\Pi_{S}} a_{n}=b
$$

which implies that

$$
\left(a_{1}, a_{2}\right), \cdots,\left(a_{n-1}, a_{n}\right) \in \Pi_{S} \subseteq\left(S \cup S^{-1}\right)^{c}=S^{c} \cup\left(S^{c}\right)^{-1} \subseteq\left(S^{c}\right)^{e}=\langle S\rangle .
$$

From the transitivity, we get $(a, b)=\left(a_{1}, a_{n}\right) \in\langle S\rangle$, as required.

Lemma 2.19. Let $X$ be a set and $\leqslant$ a monomial order on $\mathfrak{M}(X)$. Let $S$ be a binary relation on $\mathfrak{M}(X)$. For each operated congruence class $A$ of $\langle S\rangle$, we have

$$
|A \cap \operatorname{Irr}(S)| \geqslant 1 \text { and } A=\cup_{a \in A \cap \operatorname{Irr}(S)} \mathcal{P}(a) \text {, }
$$

where $\operatorname{Irr}(S):=\mathfrak{M}(X) \backslash \operatorname{Dom}\left(\Pi_{S}\right)$ is the set of all irreducible elements under $\Pi_{S}$.

Proof. Let $b \in A$. By Lemma 2.17, there is $a \in \operatorname{Irr}(S)$ such that $b \stackrel{*}{\rightarrow}_{\Pi_{S}} a$. From Lemma2.18, we have $(b, a) \in\langle S\rangle$ and so $a \in A$. Hence $a \in A \cap \operatorname{Irr}(S)$ and $|A \cap \operatorname{Irr}(S)| \geqslant 1$. Since $b \stackrel{*}{\rightarrow} \Pi_{S} a$, it follows that $b \in \mathcal{P}(a)$ and thus

$$
A \subseteq \cup_{a \in A \cap \operatorname{Irr}(S)} \mathcal{P}(a) .
$$

Conversely, for every element $a \in A$, we have $\mathcal{P}(a) \subseteq A$ by Lemma 2.18 and so

$$
\cup_{a \in A \cap \operatorname{Irr}(S)} \mathcal{P}(a) \subseteq A \text {. }
$$

This completes the proof.

Lemma 2.20. Let $X$ be a set and $\leqslant$ a monomial order on $\mathfrak{M}(X)$. Let $S$ be a binary relation on $\mathfrak{M}(X)$. For each operated congruence class $A$ of $\langle S\rangle$, if $\Pi_{S}$ is confluent, then $|A \cap \operatorname{Irr}(S)|=1$.

Proof. Assume that $\Pi_{S}$ is confluent. By Lemma 2.19, we get $|A \cap \operatorname{Irr}(S)| \geqslant 1$. Suppose to the contrary that $|A \cap \operatorname{Irr}(S)| \geqslant 2$. Let $A \cap \operatorname{Irr}(S)=\left\{a_{i} \mid i \in I\right\}$ with $|I| \geqslant 2$. We have two cases to consider.

Case 1. $\mathcal{P}\left(a_{i}\right) \cap \mathcal{P}\left(a_{j}\right) \neq \emptyset$ for some $i, j \in I$ with $i \neq j$. In this case, we can choose $b \in \mathcal{P}\left(a_{i}\right) \cap \mathcal{P}\left(a_{j}\right)$. Then $\left(b \stackrel{*}{\rightarrow} \Pi_{S} a_{i}, b \stackrel{*}{\rightarrow}_{\Pi_{S}} a_{j}\right)$ is a fork, which is not joinable by $a_{i}, a_{j} \in \operatorname{Irr}(S)$, contradicting that $\Pi_{S}$ is confluent. 
Case 2. $\mathcal{P}\left(a_{i}\right) \cap \mathcal{P}\left(a_{j}\right)=\emptyset$ for all $i, j \in I$ with $i \neq j$. We claim that $\left(t_{i}, t_{j}\right),\left(t_{j}, t_{i}\right) \notin \Pi_{S}$ for all $t_{i} \in \mathcal{P}\left(a_{i}\right)$ and $t_{j} \in \mathcal{P}\left(a_{j}\right)$. Otherwise, by symmetry, let $\left(t_{i}, t_{j}\right) \in \Pi_{S}$ for some $t_{i} \in \mathcal{P}\left(a_{i}\right)$ and $t_{j} \in \mathcal{P}\left(a_{j}\right)$. Then $t_{i} \rightarrow \Pi_{S} t_{j} \stackrel{*}{\rightarrow}_{\Pi_{S}} a_{j}$ and so $t_{i} \in \mathcal{P}\left(a_{j}\right) \cap \mathcal{P}\left(a_{i}\right)$, contradicting that $\mathcal{P}\left(a_{i}\right) \cap \mathcal{P}\left(a_{j}\right)=\emptyset$.

Since $a_{i}$ and $a_{j}$ are in the same operated congruence class $A$, we have $\left(a_{i}, a_{j}\right) \in\langle S\rangle$. By Lemma 2.11, there is a sequence $a_{i}=v_{1}, v_{2}, \cdots, v_{n}=a_{j}$ with $n \geqslant 2$, in which, for each $k$ in $\{1,2, \cdots, n-1\}$, either $\left(v_{k}, v_{k+1}\right) \in S^{c}$ or $\left(v_{k+1}, v_{k}\right) \in S^{c}$. Note that $v_{k} \in A, 1 \leqslant k \leqslant n$. Because $a_{i} \in \operatorname{Irr}(S)$, we can take $\ell:=\max \left\{k \mid v_{k} \in \mathcal{P}\left(a_{i}\right), 1 \leqslant k \leqslant n\right\}$. If $\ell=n$, then $a_{j}=v_{n} \in \mathcal{P}\left(a_{i}\right)$ and so $a_{j} \in \mathcal{P}\left(a_{i}\right) \cap \mathcal{P}\left(a_{j}\right)$, a contradiction. If $1 \leqslant \ell \leqslant n-1$, then

$$
v_{\ell} \in \mathcal{P}\left(a_{i}\right), v_{\ell+1} \notin \mathcal{P}\left(a_{i}\right) \text { and } v_{\ell} \rightarrow_{\Pi_{S}} v_{\ell+1} .
$$

Since $v_{\ell+1} \in A$, by Lemma 2.19, there exists $p \in I$ with $p \neq i$ such that $v_{\ell+1} \stackrel{*}{\rightarrow} \Pi_{S} a_{p}$. Thus $v_{\ell} \stackrel{*}{\rightarrow}_{\Pi_{S}} a_{i}$ and $v_{\ell} \rightarrow \Pi_{S} v_{\ell+1} \stackrel{*}{\rightarrow}_{\Pi_{S}} a_{p}$, but $a_{i}, a_{p} \in \operatorname{Irr}(S)$ and $a_{i} \neq a_{p}$, contradicting that $\Pi_{S}$ is confluent.

Now we are ready for our main result of this section.

Theorem 2.21. Let $S$ be a binary relation on $\mathfrak{M}(X)$, and let $\leqslant$ be a monomial order on $\mathfrak{M}(X)$ and $\Pi_{S}$ the term-rewriting system with respect to $\leqslant$. Then $\operatorname{Irr}(S)$ is a section of $\langle S\rangle$ if and only if $\Pi_{S}$ is convergent.

Proof. $(\Leftarrow)$ Suppose $\Pi_{S}$ is convergent. Then in view of Lemma 2.20, every operated congruence class intersects with $\operatorname{Irr}(S)$ exactly one element. $\operatorname{So} \operatorname{Irr}(S)$ is a section of $\mathfrak{M}(X) /\langle S\rangle$.

$(\Rightarrow)$ Suppose to the contrary that $\Pi_{S}$ is not convergent. Since $\Pi_{S}$ is terminating by Lemma2.17, $\Pi_{S}$ is not confluent. Then there exists a fork $\left(t \stackrel{*}{\rightarrow} \Pi_{S} v_{1}, t \stackrel{*}{\rightarrow} \Pi_{S} v_{2}\right)$ which is not joinable. By Lemma 2.17,

$$
v_{1} \stackrel{*}{\rightarrow}_{\Pi_{S}} u_{1} \text { and } v_{2} \stackrel{*}{\rightarrow}_{\Pi_{S}} u_{2} \text { for some } u_{1}, u_{2} \in \operatorname{Irr}(S), u_{1} \neq u_{2} .
$$

which implies that $t \stackrel{*}{\rightarrow} \Pi_{S} u_{1}$ and $t \stackrel{*}{\rightarrow} \Pi_{S} u_{2}$. By Lemma 2.18, we have

$$
\left(t, u_{1}\right),\left(t, u_{2}\right) \in\langle S\rangle \text { and so }\left(u_{1}, u_{2}\right) \in\langle S\rangle \text {. }
$$

Hence $u_{1}$ and $u_{2}$ in a same operated congruence class and they are in normal form, contradicting that $\operatorname{Irr}(S)$ is a section of $\mathfrak{M}(X) /\langle S\rangle$.

\section{Applichtions}

Inverse monoids appear in a range of contexts, for example, they can be employed in the study of partial symmetries [24]. $U$-monoids are natural generalizations of inverse monoids.

Definition 3.1. A $U$-monoid is a monoid $G$ equipped with a (unary) operator ${ }^{\circ}$ such that $\left(u^{\circ}\right)^{\circ}=u$ for all $u \in G$.

The following are two classes of $U$-monoids.

Definition 3.2. (a) A *-monoid is a $U$-monoid $G$ satisfying the axiom $(u v)^{\circ}=v^{\circ} u^{\circ}$ for all $u, v \in G$. Such an operator is called an involution, and typically denoted by $*$.

(b) A group is a $*$-monoid $G$ satisfying the axiom $u^{*} u=1=u u^{*}$ for all $u \in G$.

In this section, as applications of Theorem 2.21, we construct respectively sections of free $*-$ monoids and free groups, which are viewed as quotients of free operated monoids. The monomial order given in [18, Lem. 5.3] will be used throughout this section. 
3.1. Free $*$-monoids. This subsection is spent to construct sections of free $*$-monoids. Recall that $\mathfrak{M}(X)$ is the free operated monoid on $X$. As a special case of a well-known result in universal algebra [10, Prop.1.3.6], the free $*$-monoid on a set $X$ is the quotient of $\mathfrak{M}(X)$ by the operated congruence $\langle S\rangle$, where

$$
S=\{\phi(w):=(\lfloor\lfloor w\rfloor, w), \psi(u, v):=(\lfloor u v\rfloor,\lfloor v\rfloor\lfloor u\rfloor), \omega:=(\lfloor 1\rfloor, 1) \mid w \in \mathfrak{M}(X), u, v \in \mathfrak{M}(X) \backslash\{1\}\} .
$$

Before we go on to obtain a section of the free $*$-monoid, we recall a monomial order on $\mathfrak{M}(X)$ from [18]. Let $\leqslant$ be a well-ordering on a set $X$. It can be extended to a well-ordering on $\mathfrak{M}(X)=\lim \mathfrak{M}_{n}(X)$ by recursively defining a well-ordering $\leqslant_{n}$, on $\mathfrak{M}_{n}:=\mathfrak{M}_{n}(X)$ for each $n \geqslant 0$. When $n=\overrightarrow{0}$, we have $\mathfrak{M}_{0}=M(X)$. In this case, we obtain a well-ordering by taking the shortlex order $\leqslant_{\mathrm{s} l}$ on $M(X)$ induced by $\leqslant$ with the convention that $1 \leqslant_{\mathrm{s} l} u$ for all $u \in M(X) \backslash\{1\}$. Suppose $\leqslant_{n}$ has been defined on $\mathfrak{M}_{n}:=M\left(X \sqcup\left\lfloor\mathfrak{M}_{n-1}\right\rfloor\right)$ for an $n \geqslant 0$. Denote by $\operatorname{deg}_{X}(u)$ the number of $x \in X$ in $u$ with repetition. Then $\leqslant_{n}$ induces

(a) a well-ordering $\leqslant_{n}^{\prime}$ on $\left\lfloor\mathfrak{M}_{n}\right\rfloor$ by

$$
\lfloor u\rfloor<_{n}^{\prime}\lfloor v\rfloor \Longleftrightarrow u<_{n} v \text {; }
$$

(b) a well-ordering $\leqslant_{n}^{\prime \prime}$ on $X \sqcup\left\lfloor\mathfrak{M}_{n}\right\rfloor$;

(c) a well-ordering $\leqslant_{n}^{\prime \prime \prime}$ on $X \sqcup\left\lfloor\mathfrak{M}_{n}\right\rfloor$ by

$$
u<_{n}^{\prime \prime \prime} v \Longleftrightarrow\left\{\begin{array}{l}
\text { either } \operatorname{deg}_{X}(u)<\operatorname{deg}_{X}(v) \\
\operatorname{or}_{\operatorname{deg}_{X}}(u)=\operatorname{deg}_{X}(v) \text { and } u<_{n}^{\prime \prime} v .
\end{array}\right.
$$

(d) the shortlex well-ordering $\leqslant_{n+1}$ on $\mathfrak{M}_{n+1}=M\left(X \sqcup\left\lfloor\mathfrak{M}_{n}\right\rfloor\right)$ induced by $\leqslant_{n}^{\prime \prime \prime}$.

The orders $\leqslant_{n}$ are compatible with the direct system $\left\{\mathfrak{M}_{n}\right\}_{n \geqslant 0}$ and hence induces a well-ordering, still denoted by $\leqslant$, on $\mathfrak{M}(X)=\underset{\lim }{\longrightarrow} \mathfrak{M}_{n}$.

Lemma 3.3. [18, Lem. 5.3] The order $\leqslant$ on $\mathfrak{M}(X)$ defined above is a monomial order.

Using this monomial order, we have

$$
w<\lfloor\lfloor w\rfloor\rfloor,\lfloor v\rfloor\lfloor u\rfloor<\lfloor u v\rfloor, 1<\lfloor 1\rfloor \text { for all } w \in \mathfrak{M}(X) \text {, and } u, v \in \mathfrak{M}(X) \backslash\{1\} .
$$

For simplicity, if $\alpha$ is an element of a binary relation, we denote $\bar{\alpha}$ and $R(\alpha)$ by the domain and image of $\alpha$, respectively. For example,

$$
\overline{\phi(w)}=\lfloor\lfloor w\rfloor, R(\phi(w))=w, \overline{\psi(u, v)}=\lfloor u v\rfloor, R(\varphi(u))=\lfloor v\rfloor\lfloor\rfloor, \text { and } \bar{\omega}=\lfloor 1\rfloor, R(\omega)=1 .
$$

In the remainder of this paper, if $\left.\left.q\right|_{\lfloor\lfloor x\rfloor\rfloor} \rightarrow_{\Pi_{S}} q\right|_{x}$, we will indicate such rewriting step in more detail by $\left.\left.q\right|_{\lfloor\lfloor x\rfloor\rfloor} \rightarrow_{\phi} q\right|_{x}$. Similar notations will be used for $\rightarrow_{\varphi}$ and $\rightarrow_{\omega}$.

The following concept is finer than bracketed subwords, containing the information of placements [29].

Definition 3.4. Let $X$ be a set, and let $w \in \mathfrak{M}(X)$ be such that

$$
\left.q_{1}\right|_{u_{1}}=w=\left.q_{2}\right|_{u_{2}} \text { for some } u_{1}, u_{2} \in \mathfrak{M}(X), q_{1}, q_{2} \in \mathfrak{M}^{\star}(X) .
$$

The two placements $\left(u_{1}, q_{1}\right)$ and $\left(u_{2}, q_{2}\right)$ are called

(a) separated if there exist $p \in \mathfrak{M}^{\star_{1}, \star_{2}}(X)$ such that $\left.q_{1}\right|_{\star_{1}}=\left.p\right|_{\star_{1}, u_{2}},\left.q_{2}\right|_{\star_{2}}=\left.p\right|_{u_{1}, \star_{2}}$, and $w=\left.p\right|_{u_{1}, u_{2}}$

(b) nested if there exists $q \in \mathfrak{M}^{\star}(X)$ such that either $q_{2}=\left.q_{1}\right|_{q}$ or $q_{1}=\left.q_{2}\right|_{q}$;

(c) intersecting if there exist $q \in \mathfrak{M}^{\star}(X)$ and $a, b, c \in \mathfrak{M}(X) \backslash\{1\}$ such that $w=\left.q\right|_{a b c}$ and either

(i) $q_{1}=\left.q\right|_{\star c}$ and $q_{2}=\left.q\right|_{a \star}$; or 
(ii) $q_{1}=\left.q\right|_{a \star}$ and $q_{2}=\left.q\right|_{\star c}$.

Remark 3.5. (a) Suppose the placements $\left(u_{1}, q_{1}\right)$ and $\left(u_{2}, q_{2}\right)$ are nested. If $q_{2}=\left.q_{1}\right|_{q}$, then $\left.q_{1}\right|_{q_{u_{2}}}=\left.q_{2}\right|_{u_{2}}=w=\left.q_{1}\right|_{u_{1}}$. By Remark 2.4, we have $\left.q\right|_{u_{2}}=u_{1}$, i.e., $u_{2}$ is a bracketed subword of $u_{1}$. Similarly, if $q_{1}=\left.q_{2}\right|_{q}$, then $\left.q\right|_{u_{1}}=u_{2}$, i.e., $u_{1}$ is a bracketed subword of $u_{2}$.

(b) Suppose the placements $\left(u_{1}, q_{1}\right)$ and $\left(u_{2}, q_{2}\right)$ are intersecting. If $q_{1}=\left.q\right|_{\star c}$ and $q_{2}=\left.q\right|_{\star \star}$, then

$$
\left.q\right|_{u_{1} c}=\left.q_{1}\right|_{u_{1}}=w=\left.q\right|_{a b c} \text { and }\left.q\right|_{a u_{2}}=\left.q_{2}\right|_{u_{2}}=w=\left.q\right|_{a b c} .
$$

Again by Remark 2.4, $u_{1} c=a b c$ and $a u_{2}=a b c$. So $u_{1}=a b$ and $u_{2}=b c$. Similarly, if $q_{1}=\left.q\right|_{a \star}$ and $q_{2}=\left.q\right|_{\star c}$, then $u_{1}=a b$ and $u_{2}=b c$.

Lemma 3.6. [29, Thm. 4.11] Let $w \in \mathfrak{M}(X)$. For any two placements $\left(u_{1}, q_{1}\right)$ and $\left(u_{2}, q_{2}\right)$ in $w$, exactly one of the following is true:

(a) $\left(u_{1}, q_{1}\right)$ and $\left(u_{2}, q_{2}\right)$ are separated;

(b) $\left(u_{1}, q_{1}\right)$ and $\left(u_{2}, q_{2}\right)$ are nested;

(c) $\left(u_{1}, q_{1}\right)$ and $\left(u_{2}, q_{2}\right)$ are intersecting.

Lemma 3.7. Let $w \in \mathfrak{M}(X)$. For any two placements $\left(u_{1}, q_{1}\right)$ and $\left(u_{2}, q_{2}\right)$ in $w$, if the breadth $\left|u_{1}\right|$ is 1 , then $\left(u_{1}, q_{1}\right)$ and $\left(u_{2}, q_{2}\right)$ cann't be intersecting.

Proof. Suppose the placements $\left(u_{1}, q_{1}\right)$ and $\left(u_{2}, q_{2}\right)$ are intersecting. From Definition 3.4 (C) , there are $q \in \mathfrak{M}^{\star}(X)$ and $a, b, c \in \mathfrak{M}(X) \backslash\{1\}$ such that $w=\left.q\right|_{a b c}$ and either $q_{1}=\left.q\right|_{\star c}$ and $q_{2}=\left.q\right|_{a_{\star}}$, or $q_{1}=\left.q\right|_{a \star}$ and $q_{2}=\left.q\right|_{\star c}$. For the former case, we have $u_{1}=a b$ and $u_{2}=b c$ by Remark 3.5, for the later case, we get $u_{1}=b c$ and $u_{2}=a b$. Since $a, b, c \in \mathfrak{M}(X) \backslash\{1\}$, it follows that $|a|,|b|,|c|>1$ and so $\left|u_{1}\right| \geqslant 2$ in both cases, contradicting that $\left|u_{1}\right|=1$.

Lemma 3.8. Let $S$ be the binary relation given in $E q$. (4) and $\alpha, \beta \in S$. Suppose $\left.q_{1}\right|_{\bar{\alpha}}=\left.q_{2}\right|_{\bar{\beta}}$ for some $q_{1}, q_{2} \in \mathfrak{M}^{\star}(X)$. If the placements $\left(\bar{\alpha}, q_{1}\right)$ and $\left(\bar{\beta}, q_{2}\right)$ are separated, then $\left.\left.q_{1}\right|_{R(\alpha)} \downarrow_{\Pi_{S}} q_{2}\right|_{R(\beta)}$.

Proof. In view of Definition $3.4(\mathbb{a})$, there exists $p \in \mathfrak{M}^{\star_{1}, \star_{2}}(X)$ such that

$$
\left.q_{1}\right|_{\star_{1}}=\left.p\right|_{\star_{1}, \bar{\beta}} \text { and }\left.q_{2}\right|_{\star_{2}}=\left.p\right|_{\bar{\alpha}, \star_{2}},
$$

whence

$$
\begin{aligned}
& \left.q_{1}\right|_{R(\alpha)}=\left.\left.p\right|_{R(\alpha), \bar{\beta}} \rightarrow_{\Pi_{S}} p\right|_{R(\alpha), R(\beta)}, \\
& \left.q_{2}\right|_{R(\beta)}=\left.\left.p\right|_{\bar{\alpha}, R(\beta)} \rightarrow_{\Pi_{S}} p\right|_{R(\alpha), R(\beta)} .
\end{aligned}
$$

So we conclude that $\left.\left.q_{1}\right|_{R(\alpha)} \downarrow_{\Pi_{S}} q_{2}\right|_{R(\beta)}$.

Lemma 3.9. Let $S$ be the binary relation given in Eq. (4) and $\alpha, \beta \in S$. Suppose $\left.q_{1}\right|_{\bar{\alpha}}=\left.q_{2}\right|_{\bar{\beta}}$ for some $q_{1}, q_{2} \in \mathfrak{M}^{\star}(X)$. If the placements $\left(\bar{\alpha}, q_{1}\right)$ and $\left(\bar{\beta}, q_{2}\right)$ are nested, then $\left.\left.q_{1}\right|_{R(\alpha)} \downarrow_{\Pi_{S}} q_{2}\right|_{R(\beta)}$.

Proof. Suppose the two placements $\left(\bar{\alpha}, q_{1}\right)$ and $\left(\bar{\beta}, q_{2}\right)$ are nested. According to the choice of $\alpha$ and $\beta$, we have the following cases to consider.

Case 1. $\bar{\alpha}=\overline{\phi(u)}=\lfloor\lfloor u\rfloor\rfloor$ and $\bar{\beta}=\overline{\phi(v)}=\lfloor\lfloor v\rfloor\rfloor$. By symmetry, we may assume that $q_{1}=\left.q_{2}\right|_{q}$ for some $q \in \mathfrak{M}^{\star}(X)$. Then by Remark 3.5, $\left.q\right|_{\lfloor u\rfloor\rfloor}=\lfloor\lfloor v\rfloor\rfloor$, i.e., $\lfloor\lfloor u\rfloor\rfloor$ is a bracketed subword of $\lfloor\lfloor v\rfloor\rfloor$.

Subcase 1.1. $\lfloor\lfloor u\rfloor\rfloor=\lfloor\lfloor v\rfloor\rfloor$. Then $\alpha=\beta, q=\star$ and $q_{1}=q_{2}$. Hence $\left.q_{1}\right|_{R(\alpha)}=\left.q_{2}\right|_{R(\beta)}$ and $\left.\left.q_{1}\right|_{R(\alpha)} \downarrow_{\Pi_{S}} q_{2}\right|_{R(\beta)}$ trivially.

Subcase 1.2. $\lfloor\lfloor u\rfloor\rfloor=\lfloor v\rfloor$. Then $v=\lfloor u\rfloor,\left.q\right|_{\lfloor u\rfloor\rfloor}=\lfloor\lfloor v\rfloor\rfloor=\lfloor\lfloor\lfloor u\rfloor\rfloor\rfloor=\lfloor\star\rfloor\lfloor\lfloor u\rfloor\rfloor$ and $q=\lfloor\star\rfloor$. Hence

$$
\left.q_{1}\right|_{R(\alpha)}=\left.q_{1}\right|_{u}=\left.\left(\left.q_{2}\right|_{q}\right)\right|_{u}=\left.q_{2}\right|_{q_{u}}=\left.q_{2}\right|_{\left\lfloor\star \star \|_{u}\right.}=\left.q_{2}\right|_{u u\rfloor}=\left.q_{2}\right|_{v}=\left.q_{2}\right|_{R(\beta)}
$$

and so $\left.\left.q_{1}\right|_{R(\alpha)} \downarrow_{\Pi_{S}} q_{2}\right|_{R(\beta)}$ trivially. 
Subcase 1.3. $\lfloor\lfloor u\rfloor\rfloor$ is a bracketed subword of $v$. Then there exists $p \in \mathfrak{M}^{\star}(X)$ such that $v=\left.p\right|_{\lfloor\lfloor u\rfloor\rfloor}$ and $q=\lfloor\lfloor p\rfloor\rfloor$, which implies

$$
\begin{gathered}
\left.q_{1}\right|_{R(\alpha)}=\left.q_{1}\right|_{u}=\left.\left(\left.q_{2}\right|_{q}\right)\right|_{u}=\left.q_{2}\right|_{\left.q\right|_{u}}=\left.q_{2}\right|_{\left\lfloor\lfloor p\rfloor \|_{u}\right.}=\left.\left.q_{2}\right|_{\left\lfloor\left\lfloor\left. p\right|_{u}\right\rfloor\right\rfloor} \rightarrow_{\phi} q_{2}\right|_{\left.p\right|_{u}}, \\
\left.q_{2}\right|_{R(\beta)}=\left.q_{2}\right|_{v}=\left.\left.q_{2}\right|_{\left.p\right|_{\lfloor\lfloor u\rfloor}} \rightarrow_{\phi} q_{2}\right|_{\left.p\right|_{u}} .
\end{gathered}
$$

Consequently $\left.\left.q_{1}\right|_{R(\alpha)} \downarrow_{\Pi_{S}} q_{2}\right|_{R(\beta)}$.

Case 2. $\bar{\alpha}=\overline{\psi\left(u^{\prime}, v^{\prime}\right)}=\left\lfloor u^{\prime} v^{\prime}\right\rfloor$ and $\bar{\beta}=\overline{\psi(u, v)}=\lfloor u v\rfloor$. By symmetry, we may assume that $q_{1}=\left.q_{2}\right|_{q}$ for some $q \in \mathfrak{M}^{\star}(X)$. Then by Remark 3.5, $\left.q\right|_{\left\lfloor u^{\prime} v^{\prime}\right\rfloor}=\lfloor u v\rfloor$, i.e., $\left\lfloor u^{\prime} v^{\prime}\right\rfloor$ is a bracketed subword of $\lfloor u v\rfloor$.

Subcase 2.1. $\left\lfloor u^{\prime} v^{\prime}\right\rfloor=\lfloor u v\rfloor$. This is similar to Subcase 1.1 .

Subcase 2.2. $\left\lfloor u^{\prime} v^{\prime}\right\rfloor$ is a bracketed subword of $u$ or $v$. By symmetry, we may assume $\left\lfloor u^{\prime} v^{\prime}\right\rfloor$ is a bracketed subword of $u$. i.e., there exists $p \in \mathfrak{M}^{\star}(X)$ such that $u=\left.p\right|_{\left\lfloor u^{\prime} v^{\prime}\right\rfloor}$ and $q=\lfloor p v\rfloor$. Whence

$$
\begin{aligned}
& \left.q_{1}\right|_{R(\alpha)}=\left.q_{1}\right|_{\left.\left\lfloor v^{\prime}\right\rfloor u^{\prime}\right\rfloor}=\left.\left(\left.q_{2}\right|_{q}\right)\right|_{\left\lfloor v^{\prime}\right\rfloor\left\lfloor u^{\prime}\right\rfloor}=\left.q_{2}\right|_{\left.\left.\left.q\right|_{\left\lfloor v^{\prime}\right.}\right\rfloor u^{\prime}\right\rfloor}=\left.q_{2}\right|_{\lfloor p v\rfloor_{\left.\left\lfloor v^{\prime}\right\rfloor u^{\prime}\right\rfloor}}=\left.\left.q_{2}\right|_{\left\lfloor\left. p\right|_{\left.\left\lfloor v^{\prime}\right\rfloor u^{\prime}\right\rfloor}\right\rfloor} \rightarrow_{\psi} q_{2}\right|_{\lfloor v\rfloor\left\lfloor p \mid\left\lfloor_{\left.\left\lfloor v^{\prime}\right\rfloor u^{\prime}\right\rfloor}\right\rfloor\right.}, \\
& \left.q_{2}\right|_{R(\beta)}=\left.q_{2}\right|_{\lfloor v\rfloor\lfloor u\rfloor}=\left.\left.q_{2}\right|_{\lfloor v\rfloor\left\lfloor p \|_{\left\lfloor u^{\prime} v^{\prime}\right\rfloor}\right.} \rightarrow{ }_{\psi} q_{2}\right|_{\lfloor v\rfloor\left\lfloor p \|_{\left.\left\lfloor v^{\prime}\right\rfloor u^{\prime}\right\rfloor}\right.}=\left.q_{2}\right|_{\llcorner v\rfloor\left\lfloor\left. p\right|_{\left\lfloor v^{\prime}\right\rfloor\left\lfloor u^{\prime}\right\rfloor}\right\rfloor} .
\end{aligned}
$$

Hence $\left.\left.q_{1}\right|_{R(\alpha)} \downarrow_{\Pi_{S}} q_{2}\right|_{R(\beta)}$.

Case 3. $\bar{\alpha}=\overline{\phi(w)}=\lfloor\lfloor w\rfloor\rfloor$ and $\bar{\beta}=\overline{\psi(u, v)}=\lfloor u v\rfloor$.

Subcase 3.1. $q_{1}=\left.q_{2}\right|_{q}$ for some $q \in \mathfrak{M}^{\star}(X)$. By Remark 3.5, $\left.q\right|_{\lfloor\lfloor w\rfloor\rfloor}=\lfloor u v\rfloor$, that is, $\lfloor\lfloor w\rfloor\rfloor$ is a bracketed subword of $\lfloor u v\rfloor$.

Subcase 3.1.1. $\lfloor\lfloor w\rfloor\rfloor=\lfloor u v\rfloor$. Then $\lfloor w\rfloor=u v$ and so $u=1$ or $v=1$, which contradicts from Eq. (6) that $\beta \in S$.

Subcase 3.1.2. $\lfloor\lfloor w\rfloor\rfloor$ is a bracketed subword of $u v$. By symmetry, we can assume that $\lfloor\lfloor w\rfloor\rfloor$ is a bracketed subword of $u$, i.e., there exists $p \in \mathfrak{M}^{\star}(X)$ such that $u=\left.p\right|_{\lfloor\lfloor w\rfloor}$ and $q=\lfloor p v\rfloor$. So

Thus $\left.\left.q_{1}\right|_{R(\alpha)} \downarrow_{\Pi_{S}} q_{2}\right|_{R(\beta)}$.

$$
\begin{gathered}
\left.q_{1}\right|_{R(\alpha)}=\left.q_{1}\right|_{w}=\left.\left(\left.q_{2}\right|_{q}\right)\right|_{w}=\left.q_{2}\right|_{q_{w}}=\left.q_{2}\right|_{\lfloor p v\rfloor_{w}}=\left.\left.q_{2}\right|_{\left\lfloor\left. p\right|_{w} v\right\rfloor} \rightarrow_{\psi} q_{2}\right|_{\lfloor v\rfloor\left\lfloor\left. p\right|_{w}\right\rfloor}, \\
\left.q_{2}\right|_{R(\beta)}=\left.q_{2}\right|_{\lfloor v\rfloor\lfloor u\rfloor}=\left.\left.q_{2}\right|_{\lfloor v\rfloor\left\lfloor\left. p\right|_{\lfloor w\rfloor\rfloor}\right\rfloor} \rightarrow{ }_{\phi} q_{2}\right|_{\lfloor v\rfloor\left\lfloor\left. p\right|_{w}\right\rfloor} .
\end{gathered}
$$

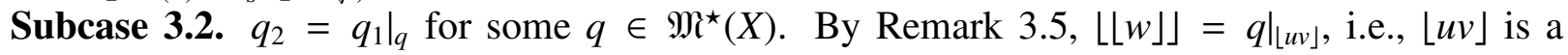
bracketed subword of $\lfloor\lfloor w\rfloor\rfloor$. If $\lfloor u v\rfloor=\lfloor\lfloor w\rfloor\rfloor$, similar to Case 3.1.1, we get $\left.\left.q_{1}\right|_{R(\alpha)} \downarrow_{\Pi_{S}} q_{2}\right|_{R(\beta)}$. Suppose $\lfloor u v\rfloor \neq\lfloor\lfloor w\rfloor\rfloor$.

Subcase 3.2.1. $\lfloor u v\rfloor=\lfloor w\rfloor$. Then

$$
u v=w,\left.q\right|_{\lfloor u v\rfloor}=\lfloor\lfloor w\rfloor\rfloor=\lfloor\lfloor v v\rfloor=\lfloor\star\rfloor\rfloor_{\lfloor u v\rfloor} \text { and } q=\lfloor\star\rfloor .
$$

Consequently $\left.q_{1}\right|_{R(\alpha)}=\left.q_{1}\right|_{w}=\left.q_{1}\right|_{u v}$ and

$$
\begin{aligned}
\left.q_{2}\right|_{R(\beta)} & =\left.q_{2}\right|_{\lfloor v\rfloor\lfloor u\rfloor}=\left.\left(\left.q_{1}\right|_{q}\right)\right|_{\lfloor v\rfloor\lfloor u\rfloor}=\left.q_{1}\right|_{\left.q\right|_{\lfloor v\rfloor u\rfloor}}=\left.q_{1}\right|_{\lfloor\star\rfloor\rfloor_{\lfloor v \downarrow u\rfloor}}=\left.q_{1}\right|_{\lfloor\lfloor v\rfloor\lfloor u\rfloor\rfloor} \\
& \left.\left.\left.\rightarrow{ }_{\psi} q_{1}\right|_{\lfloor\lfloor u\rfloor\rfloor\lfloor v\rfloor\rfloor} \rightarrow{ }_{\phi} q_{1}\right|_{u\lfloor\lfloor v\rfloor\rfloor} \rightarrow{ }_{\phi} q_{1}\right|_{u v v} .
\end{aligned}
$$

Hence $\left.\left.q_{1}\right|_{R(\alpha)} \downarrow_{\Pi_{S}} q_{2}\right|_{R(\beta)}$.

Subcase 3.2.2. $\lfloor u v\rfloor$ is a bracketed subword of $w$, i.e., there exists $p \in \mathfrak{M}^{\star}(X)$ such that $w=\left.p\right|_{\lfloor u v\rfloor}$ and $q=\lfloor\lfloor p\rfloor\rfloor$. Thus

$$
\begin{aligned}
& \left.q_{1}\right|_{R(\alpha)}=\left.q_{1}\right|_{w}=\left.\left.q_{1}\right|_{\left.p\right|_{\lfloor u v\rfloor}} \rightarrow_{\psi} q_{1}\right|_{\left.\left.p\right|_{\lfloor v\rfloor u}\right\rfloor}, \\
& \left.q_{2}\right|_{R(\beta)}=\left.q_{2}\right|_{\lfloor v\rfloor\lfloor u\rfloor}=\left.\left(\left.q_{1}\right|_{q}\right)\right|_{\lfloor v\rfloor\lfloor u\rfloor}=\left.q_{1}\right|_{\left.q\right|_{\llcorner v\lrcorner\lfloor u\rfloor}}=\left.q_{1}\right|_{\lfloor\lfloor p\rfloor\rfloor \|_{\lfloor v\rfloor u\rfloor}}=\left.\left.q_{1}\right|_{\left\lfloor\left\lfloor\left. p\right|_{\lfloor v\rfloor u u\rfloor\rfloor} \rightarrow_{\phi}\right.\right.} q_{1}\right|_{\left.\left.p\right|_{\lfloor v \downarrow\lfloor u\rfloor}\right\rfloor},
\end{aligned}
$$

and so $\left.\left.q_{1}\right|_{R(\alpha)} \downarrow_{\Pi_{S}} q_{1}\right|_{R(\beta)}$.

Case 4. $\bar{\alpha}=\overline{\phi(w)}=\lfloor\lfloor w\rfloor\rfloor$ and $\bar{\beta}=\bar{\omega}=\lfloor 1\rfloor$.

Subcase 4.1. $q_{1}=\left.q_{2}\right|_{q}$ for some $q \in \mathfrak{M}^{\star}(X)$. Then by Remark 3.5, $\left.q\right|_{\lfloor\lfloor w\rfloor\rfloor}=\lfloor 1\rfloor$, which implies that $\lfloor\lfloor w\rfloor\rfloor$ is a bracketed subword of $\lfloor 1\rfloor$, a contradiction by comparing the depth. 
Subcase 4.2. $q_{2}=\left.q_{1}\right|_{q}$ for some $q \in \mathfrak{M}^{\star}(X)$. By Remark 3.5, $\lfloor\lfloor w\rfloor\rfloor=\left.q\right|_{\lfloor 1\rfloor}$, i.e., $\lfloor 1\rfloor$ is a bracketed subword of $\lfloor\lfloor w\rfloor\rfloor$. Note that $\lfloor 1\rfloor \neq\lfloor\lfloor w\rfloor\rfloor$.

Subcase 4.2.1. $\lfloor 1\rfloor=\lfloor w\rfloor$. Then $w=1,\left.q\right|_{\lfloor 1\rfloor}=\lfloor\lfloor w\rfloor\rfloor=\left.\lfloor\star\rfloor\right|_{\lfloor 1\rfloor}$ and $q=\lfloor\star\rfloor$. Hence

$$
\begin{gathered}
\left.q_{1}\right|_{R(\alpha)}=\left.q_{1}\right|_{w}=\left.q_{1}\right|_{1}, \\
\left.q_{2}\right|_{R(\beta)}=\left.q_{2}\right|_{1}=\left.\left(\left.q_{1}\right|_{q}\right)\right|_{1}=\left.q_{1}\right|_{\left\lfloor\left.\star\right|_{1}\right.}=\left.\left.q_{1}\right|_{\lfloor 1\rfloor} \rightarrow{ }_{\omega} q_{1}\right|_{1},
\end{gathered}
$$

and so $\left.\left.q_{1}\right|_{R(\alpha)} \downarrow_{\Pi_{S}} q_{2}\right|_{R(\beta)}$.

Subcase 4.2.2. $\lfloor 1\rfloor$ is a bracketed subword of $w$, i.e., there exists $p \in \mathfrak{M}^{\star}(X)$ such that $w=\left.p\right|_{\lfloor 1\rfloor}$ and $q=\lfloor\lfloor p\rfloor\rfloor$. Then

$$
\begin{aligned}
& \left.q_{1}\right|_{R(\alpha)}=\left.q_{1}\right|_{w}=\left.\left.q_{1}\right|_{\left.p\right|_{\lfloor 1]}} \rightarrow_{\omega} q_{1}\right|_{\left.p\right|_{1}}, \\
& \left.q_{2}\right|_{R(\beta)}=\left.q_{2}\right|_{1}=\left.\left(\left.q_{1}\right|_{q}\right)\right|_{1}=\left.\left.q_{1}\right|_{\lfloor\lfloor p\rfloor\rfloor_{1}} \rightarrow{ }_{\phi} q_{1}\right|_{\left.p\right|_{1}} .
\end{aligned}
$$

Therefore $\left.\left.q_{1}\right|_{R(\alpha)} \downarrow_{\Pi_{S}} q_{1}\right|_{R(\beta)}$.

Case 5. $\bar{\alpha}=\overline{\psi(u, v)}=\lfloor u v\rfloor$ and $\bar{\beta}=\bar{\omega}=\lfloor 1\rfloor$. This is similar to Case 4 .

Case 6. $\bar{\alpha}=\bar{\omega}=\lfloor 1\rfloor$ and $\bar{\beta}=\bar{\omega}=\lfloor 1\rfloor$. This case is trivial since $\alpha$ and $\beta$ are equal.

This completes the proof.

Now we arrive at our first main result of this section.

Theorem 3.10. Let $X$ be a set and $S$ the binary relation given in Eq. (4). With the monomial order $\leqslant$ given in [18], we have

(a) the term-rewriting system $\Pi_{S}$ is convergent.

(b) the set $\operatorname{Irr}(S)=\mathfrak{M}(X) \backslash \operatorname{Dom}\left(\Pi_{S}\right)$ is a section of $\mathfrak{M}(X) /\langle S\rangle$.

Proof. (a) Since $\leqslant$ is a monomial order on $\mathfrak{M}(X), \Pi_{S}$ is terminating by Lemma 2.17, So we are left to prove that $\Pi_{S}$ is confluent. From Lemma 2.16, it suffices to show that $\Pi_{S}$ is locally confluent. Let

$$
\left.\left.q_{1}\right|_{R(\alpha) \Pi_{S}} \leftarrow q_{1}\right|_{\bar{\alpha}}=w=\left.\left.q_{2}\right|_{\bar{\beta}} \rightarrow_{\Pi_{S}} q_{2}\right|_{R(\beta)}
$$

be an arbitrary local fork, where $q_{1}, q_{2} \in \mathfrak{M}^{\star}(X), \alpha, \beta \in S$. From Eq. (4), both of the breadth of $\bar{\alpha}$ and $\bar{\beta}$ are 1 , and so the placements $\left(\bar{\alpha}, q_{1}\right)$ and $\left(\bar{\beta}, q_{2}\right)$ cann't be intersecting by Lemma 3.7. If the placements $\left(\bar{\alpha}, q_{1}\right)$ and $\left(\bar{\beta}, q_{2}\right)$ are separated and nested, then $\left.\left.q_{1}\right|_{R(\alpha)} \downarrow_{\Pi_{S}} q_{2}\right|_{R(\beta)}$ by Lemmas 3.12 and 3.9 .

(b) It follows from Item (a) and Theorem 2.21

Remark 3.11. It is well known [24] that *-monoid is also called monoid with involution, and the free monoid with involution $*$ on a set $X$ is the free monoid $M\left(X \cup X^{*}\right)$, where $X^{*}:=\left\{x^{*} \mid x \in X\right\}$ is a disjoint copy of $X$. The $\operatorname{Irr}(S)$ obtained in Theorem 3.10 is precisely the set $M\left(X \cup X^{*}\right)$ if we identify $\lfloor x\rfloor$ with $x^{*}$ for each $x \in X$.

3.2. Free groups. Again as a special case of a well-known result in universal algebra [10, Prop.1.3.6], the free group on a set $X$ is the quotient of $\mathfrak{M}(X)$ by the operated congruence $\langle S\rangle$, where

$$
S:=\{(\lfloor\lfloor w\rfloor, w),(\lfloor u v\rfloor,\lfloor v\rfloor\lfloor u),(\lfloor w\rfloor w, 1),(w\lfloor w\rfloor, 1) \mid w \in \mathfrak{M}(X), u, v \in \mathfrak{M}(X) \backslash\{1\}\} .
$$

In this subsection, we turn to construct a section of the free group on a set $W$. Write

$$
\phi(w):=(\lfloor\lfloor w\rfloor\rfloor, w), \psi(u, v):=(\lfloor u v\rfloor,\lfloor v\rfloor\lfloor u\rfloor), \varphi(w):=(\lfloor w\rfloor w, 1), \chi(w):=(w\lfloor w\rfloor, 1),
$$


where $w \in \mathfrak{M}(X), u, v \in \mathfrak{M}(X) \backslash\{1\}$. Note that if $w=1$, then $w\lfloor w\rfloor=1\lfloor 1\rfloor=\lfloor 1\rfloor$. So $(\lfloor 1\rfloor, 1) \in S$. Here again under the monomial order $\leqslant$ given in [18], we have

$$
w<\lfloor\lfloor\rfloor\rfloor,\lfloor v\rfloor\lfloor u\rfloor<\lfloor u v\rfloor, 1<\lfloor w\rfloor w, 1<w\lfloor w\rfloor \text { for } w \in \mathfrak{M}(X), u, v \in \mathfrak{M}(X) \backslash\{1\} .
$$

Lemma 3.12. Let $S$ be the binary relation given in Eq. (6) and $\alpha, \beta \in S$. Suppose $\left.q_{1}\right|_{\bar{\alpha}}=\left.q_{2}\right|_{\bar{\beta}}$ for some $q_{1}, q_{2} \in \mathfrak{M}^{\star}(X)$. If the placements $\left(\bar{\alpha}, q_{1}\right)$ and $\left(\bar{\beta}, q_{2}\right)$ are separated, then $\left.\left.q_{1}\right|_{R(\alpha)} \downarrow_{\Pi_{S}} q_{2}\right|_{R(\beta)}$.

Proof. It is parallel to the proof of Lemma 3.12, because the proof of Lemma 3.12 does not depend on the concrete expressions of $\alpha$ and $\beta$.

Lemma 3.13. Let $S$ be the binary relation given in Eq. (6) and $\alpha, \beta \in S$. Suppose $\left.q_{1}\right|_{\bar{\alpha}}=\left.q_{2}\right|_{\bar{\beta}}$ for some $q_{1}, q_{2} \in \mathfrak{M}^{\star}(X)$. If the placements $\left(\bar{\alpha}, q_{1}\right)$ and $\left(\bar{\beta}, q_{2}\right)$ are intersecting, then $\left.\left.q_{1}\right|_{R(\alpha)} \downarrow_{\Pi_{S}} q_{2}\right|_{R(\beta)}$.

Proof. Note that $\overline{\phi(w)}=\lfloor\lfloor w\rfloor\rfloor, \overline{\psi(u, v)}=\lfloor u v\rfloor$ and $|\overline{\phi(u)}|=|\overline{\psi(u, v)}|=1$ for all $w \in \mathfrak{M}(X), u, v \in$ $\mathfrak{M}(X) \backslash\{1\}$. It follows from Lemma 3.7 that $\alpha, \beta \in\{\varphi(u), \chi(u) \mid u \in \mathfrak{M}(X)\}$. Let $w:=\left.q_{1}\right|_{\bar{\alpha}}=\left.q_{2}\right|_{\bar{\beta}}$. Then by Definition 3.4( $(\mathcal{C})$, there are $q \in \mathfrak{M}^{\star}(X)$ and $a, b, c \in \mathfrak{M}(X) \backslash\{1\}$ such that $w=\left.q\right|_{a b c}$. Depending on the forms of $\alpha$ and $\beta$, there are three cases to consider.

Case 1. $\bar{\alpha}=\overline{\varphi(u)}=\lfloor u\rfloor u$ and $\bar{\beta}=\overline{\varphi(v)}=\lfloor v\rfloor v$ for some $u, v \in \mathfrak{M}(X)$. By symmetry, we may assume $q_{1}=\left.q\right|_{\star c}$ and $q_{2}=\left.q\right|_{a \star}$. Using Remark 3.5, we get $\lfloor u\rfloor u=a b$ and $\lfloor v\rfloor v=b c$. Since $|b| \geqslant 1$, $\lfloor v\rfloor$ is a bracketed subword of $b$. Suppose $v=v_{1} v_{2}$ and $b=\lfloor v\rfloor v_{1}$. Then $c=v_{2}$. Similarly from $\lfloor u\rfloor u=a b$, we can assume $a=\lfloor u\rfloor u_{1}$ and $b=u_{2}$ with $u=u_{1} u_{2}$. Then

$$
u_{2}=b=\lfloor v\rfloor v_{1}=\left\lfloor v_{1} v_{2}\right\rfloor v_{1} \text { and } a=\lfloor u\rfloor u_{1}=\left\lfloor u_{1} u_{2}\right\rfloor u_{1}=\left\lfloor u_{1}\left\lfloor v_{1} v_{2}\right\rfloor v_{1}\right\rfloor u_{1}
$$

and so

$$
\begin{aligned}
& \left.q_{1}\right|_{R(\alpha)}=\left.q_{1}\right|_{1}=\left.q\right|_{\left.\star c\right|_{1}}=\left.q\right|_{c}=\left.q\right|_{v_{2}}, \\
& \left.q_{2}\right|_{R(\beta)}=\left.q_{2}\right|_{1}=\left.q\right|_{\left.a \star\right|_{1}}=\left.q\right|_{a}=\left.\left.q\right|_{\left\lfloor u_{1}\left\lfloor v_{1} v_{2}\right\rfloor v_{1}\right\rfloor u_{1}} \rightarrow_{\psi} q\right|_{\left\lfloor\left\lfloor v_{1} v_{2}\right\rfloor v_{1}\right\rfloor\left\lfloor u_{1}\right\rfloor u_{1}} \\
& \left.\left.\left.\left.\quad \rightarrow_{\varphi} q\right|_{\left\lfloor\left\lfloor v_{1} v_{2}\right\rfloor v_{1}\right\rfloor} \rightarrow_{\psi} q\right|_{\left\lfloor\left\lfloor v_{2}\right\rfloor\left\lfloor v_{1}\right\rfloor v_{1}\right\rfloor} \rightarrow_{\varphi} q\right|_{\left\lfloor\left\lfloor v_{2}\right\rfloor\right\rfloor} \rightarrow_{\phi} q\right|_{v_{2}},
\end{aligned}
$$

which implies $\left.\left.q_{1}\right|_{R(\alpha)} \downarrow_{\Pi_{S}} q_{2}\right|_{R(\beta)}$.

Case 2. $\bar{\alpha}=\overline{\chi(u)}=u\lfloor u\rfloor$ and $\bar{\beta}=\overline{\chi(v)}=v\lfloor v\rfloor$ for some $u, v \in \mathfrak{M}(X)$. This is similar to Case 1 .

Case 3. $\bar{\alpha}=\overline{\varphi(u)}=\lfloor u\rfloor u$ and $\bar{\beta}=\overline{\chi(v)}=v\lfloor v\rfloor$, or $\bar{\alpha}=\overline{\chi(u)}=u\lfloor u\rfloor$ and $\bar{\beta}=\overline{\varphi(v)}=\lfloor v\rfloor v$ for some $u, v \in \mathfrak{M}(X)$. By symmetry, it suffices to consider the former case. Then according to Definition 3.4( $(\mathcal{C})$, we have two subcases to consider.

Subcase 3.1. $q_{1}=\left.q\right|_{\star c}$ and $q_{2}=\left.q\right|_{a \star}$. From Remark 3.5, $\lfloor u\rfloor u=a b$ and $v\lfloor v\rfloor=b c$. With a similar argument to Case 1, we can assume

$$
\begin{aligned}
& b=v_{1}, c=v_{2}\left\lfloor v_{1} v_{2}\right\rfloor \text { with } v=v_{1} v_{2}, \\
& a=\left\lfloor u_{1} u_{2}\right\rfloor u_{1}, b=u_{2} \text { with } u=u_{1} u_{2} .
\end{aligned}
$$

Thus

$$
\begin{aligned}
& \left.q_{1}\right|_{R(\alpha)}=\left.q_{1}\right|_{1}=\left.q\right|_{\left.\star c\right|_{1}}=\left.q\right|_{c}=\left.\left.\left.q\right|_{v_{2}\left\lfloor v_{1} v_{2}\right\rfloor} \rightarrow_{\psi} q\right|_{v_{2}\left\lfloor v_{2}\right\rfloor\left\lfloor v_{1}\right\rfloor} \rightarrow_{\chi} q\right|_{\left\lfloor v_{1}\right\rfloor}, \\
& \left.q_{2}\right|_{R(\beta)}=\left.q_{2}\right|_{1}=\left.q\right|_{\left.a \star\right|_{1}}=\left.q\right|_{a}=\left.\left.\left.q\right|_{\left\lfloor u_{1} u_{2}\right\rfloor u_{1}} \rightarrow_{\psi} q\right|_{\left\lfloor u_{2}\right\rfloor\left\lfloor u_{1}\right\rfloor u_{1}} \rightarrow_{\varphi} q\right|_{\left\lfloor u_{2}\right\rfloor}=\left.q\right|_{\left\lfloor v_{1}\right\rfloor},
\end{aligned}
$$

and so $\left.\left.q_{1}\right|_{R(\alpha)} \downarrow_{\Pi_{S}} q_{2}\right|_{R(\beta)}$.

Subcase 3.2. $q_{1}=\left.q\right|_{a \star}$ and $q_{2}=\left.q\right|_{\star c}$. From Remark 3.5, $\lfloor u\rfloor u=b c$ and $v\lfloor v\rfloor=a b$. Again similar to Case 1 , we may suppose

$$
b=\left\lfloor u_{1} u_{2}\right\rfloor u_{1} \text { and } c=u_{2} \text { with } u=u_{1} u_{2} .
$$


Then $a\left\lfloor u_{1} u_{2}\right\rfloor u_{1}=a b=v\lfloor v\rfloor$. If $u_{1} \neq 1$, then $\lfloor v\rfloor$ is a bracketed subword of $u_{1}$ and $a\left\lfloor u_{1} u_{2}\right\rfloor$ is a bracketed subword of $v$. So we get

$$
\operatorname{dep}(\lfloor v\rfloor) \leqslant \operatorname{dep}\left(u_{1}\right)<\operatorname{dep}\left(a\left\lfloor u_{1} u_{2}\right\rfloor\right) \leqslant \operatorname{dep}(v)
$$

a contradiction. So

$$
u_{1}=1, u_{2}=u, b=\left\lfloor u_{1} u_{2}\right\rfloor=\lfloor u\rfloor, c=u, a\lfloor u\rfloor=a b=v\lfloor v\rfloor,
$$

which implies that $a=v,\lfloor u\rfloor=\lfloor v\rfloor$ and $u=v$. Thus

$$
\begin{array}{r}
\left.q_{1}\right|_{R(\alpha)}=\left.q_{1}\right|_{1}=\left.q\right|_{\left.a \star\right|_{1}}=\left.q\right|_{a}=\left.q\right|_{v}, \\
\left.q_{2}\right|_{R(\beta)}=\left.q_{2}\right|_{1}=\left.q\right|_{\left.\star c\right|_{1}}=\left.q\right|_{c}=\left.q\right|_{u}=\left.q\right|_{v},
\end{array}
$$

and so $\left.\left.q_{1}\right|_{R(\alpha)} \downarrow_{\Pi_{S}} q_{2}\right|_{R(\beta)}$.

Lemma 3.14. Let $S$ be the binary relation given in Eq. (6) and $\alpha, \beta \in S$. Suppose $\left.q_{1}\right|_{\bar{\alpha}}=\left.q_{2}\right|_{\bar{\beta}}$ for some $q_{1}, q_{2} \in \mathfrak{M}^{\star}(X)$. If the placements $\left(\bar{\alpha}, q_{1}\right)$ and $\left(\bar{\beta}, q_{2}\right)$ are nested, then $\left.\left.q_{1}\right|_{R(\alpha)} \downarrow_{\Pi_{S}} q_{2}\right|_{R(\beta)}$.

Proof. By Definition 3.4(C), we can assume $\left.q_{1}\right|_{\bar{\alpha}}=w=\left.q_{2}\right|_{\bar{\beta}}$ for some $w \in \mathfrak{M}(X)$. From Eq. (6), there are four choices for each $\alpha$ and $\beta$. In view of symmetry, there are ten pairs of $\alpha$ and $\beta$ to consider. If $\alpha, \beta \in\{\phi(w), \psi(u, v) \mid w \in \mathfrak{M}(X), u, v \in \mathfrak{M}(X) \backslash\{1\}\}$, the result follows from Lemma 3.9. So three cases have been done and we are left to consider the following seven cases. Case 1. $\bar{\alpha}=\phi(u)=\lfloor\lfloor u\rfloor$ and $\bar{\beta}=\varphi(v)=\lfloor v\rfloor v$. Since either $\bar{\alpha}$ is subword of $\bar{\beta}$ or $\bar{\beta}$ is subword of $\bar{\alpha}$, we have the following two subcases.

Subcase 1.1. $q_{2}=\left.q_{1}\right|_{q}$ for some $q \in \mathfrak{M}^{\star}(X)$. By Remark 3.5 $\lfloor\lfloor u\rfloor\rfloor=\left.q\right|_{\lfloor v\rfloor v}$, i.e., $\lfloor v\rfloor v$ is a bracketed subword of $\lfloor\lfloor u\rfloor\rfloor$. Note that $\lfloor v\rfloor v \neq\lfloor\lfloor u\rfloor\rfloor,\lfloor u\rfloor$ by comparing the breadth. So $\lfloor v\rfloor v$ is a bracketed subword of $u$, i.e., there exists $p \in \mathfrak{M}^{\star}(X)$ such that $u=\left.p\right|_{\lfloor v\rfloor v}$ and $q=\lfloor\lfloor p\rfloor\rfloor$. Thus

$$
\begin{aligned}
& \left.q_{1}\right|_{R(\alpha)}=\left.q_{1}\right|_{u}=\left.\left.q_{1}\right|_{\left.p\right|_{\lfloor\downarrow \jmath v}} \rightarrow_{\varphi} q_{1}\right|_{\left.p\right|_{1}}, \\
& \left.q_{2}\right|_{R(\beta)}=\left.q_{2}\right|_{1}=\left.q_{1}\right|_{\left.q\right|_{1}}=\left.q_{1}\right|_{\left\lfloor\lfloor p\rfloor \|_{1}\right.}=\left.\left.q_{1}\right|_{\left\lfloor\left\lfloor\left. p\right|_{1}\right\rfloor\right\rfloor} \rightarrow_{\phi} q_{1}\right|_{\left.p\right|_{1}},
\end{aligned}
$$

and so $\left.\left.q_{1}\right|_{R(\alpha)} \downarrow_{\Pi_{S}} q_{2}\right|_{R(\beta)}$.

Subcase 1.2. $q_{1}=\left.q_{2}\right|_{q}$ for some $q \in \mathfrak{M}^{\star}(X)$. Then by Remark 3.5, $\left.q\right|_{\lfloor\lfloor u\rfloor\rfloor}=\lfloor v\rfloor v$, i.e., $\lfloor\lfloor u\rfloor\rfloor$ is a bracketed subword of $\lfloor v\rfloor v$. Note that $\lfloor\lfloor u\rfloor\rfloor\lfloor v\rfloor v$. So there are two points to consider.

Subcase 1.2.1. $\lfloor\lfloor u\rfloor\rfloor=\lfloor v\rfloor$. Then $v=\lfloor u\rfloor$. Since $\left.q\rfloor_{\lfloor\lfloor u\rfloor\rfloor}=\lfloor v\rfloor v=\lfloor\lfloor u\rfloor\rfloor u\right\rfloor$, we have $q=\star\lfloor u\rfloor$. Thus

$$
\left.q_{1}\right|_{R(\alpha)}=\left.q_{1}\right|_{u}=\left.q_{2}\right|_{\left.q\right|_{u}}=\left.\left.q_{2}\right|_{u\lfloor u\rfloor} \rightarrow_{\chi} q_{2}\right|_{1} \text { and }\left.q_{2}\right|_{R(\beta)}=\left.q_{2}\right|_{1}
$$

and so $\left.\left.q_{1}\right|_{R(\alpha)} \downarrow_{\Pi_{S}} q_{2}\right|_{R(\beta)}$.

Subcase 1.2.2. $\lfloor\lfloor u\rfloor\rfloor$ is a bracketed subword of $v$, i.e., there exists $p \in \mathfrak{M}^{\star}(X)$ such that $v=\left.p\right|_{\lfloor\lfloor u\rfloor\rfloor}$. Then $q=\lfloor p\rfloor v$ or $q=\lfloor v\rfloor p$. If $q=\lfloor p\rfloor v$, then

$$
\left.q_{1}\right|_{R(\alpha)}=\left.q_{1}\right|_{u}=\left.q_{2}\right|_{\left.q\right|_{u}}=\left.q_{2}\right|_{\left\lfloor\left. p\right|_{u}\right\rfloor v}=\left.\left.\left.q_{2}\right|_{\left.\left\lfloor\left. p\right|_{u}\right\rfloor p\right|_{\lfloor u\rfloor\rfloor}} \rightarrow_{\phi} q_{2}\right|_{\left.\left\lfloor\left. p\right|_{u}\right\rfloor p\right|_{u}} \rightarrow_{\varphi} q_{2}\right|_{1} .
$$

If $q=\lfloor v\rfloor p$, then

$$
\left.q_{1}\right|_{R(\alpha)}=\left.q_{1}\right|_{u}=\left.q_{2}\right|_{\left.q\right|_{u}}=\left.q_{2}\right|_{\left.\llcorner v\rfloor p\right|_{u}}=\left.\left.\left.q_{2}\right|_{\left.\left\lfloor\left. p\right|_{\lfloor\lfloor u\rfloor\rfloor}\right\rfloor p\right|_{u}} \rightarrow_{\phi} q_{2}\right|_{\left.\left\lfloor\left. p\right|_{u}\right\rfloor p\right|_{u}} \rightarrow_{\varphi} q_{2}\right|_{1} .
$$

Note that $\left.q_{2}\right|_{R(\beta)}=\left.q_{2}\right|_{1}$. So we conclude $\left.\left.q_{1}\right|_{R(\alpha)} \downarrow_{\Pi_{S}} q_{2}\right|_{R(\beta)}$.

Case 2. $\bar{\alpha}=\phi(u)=\lfloor\lfloor u\rfloor\rfloor$ and $\bar{\beta}=\chi(v)=v\lfloor v\rfloor$. This is similar to Case 1 .

Case 3. $\bar{\alpha}=\psi\left(u_{1}, u_{2}\right)=\left\lfloor u_{1} u_{2}\right\rfloor$ and $\bar{\beta}=\varphi(v)=\lfloor v\rfloor v$. Again since either $\bar{\alpha}$ is subword of $\bar{\beta}$ or $\bar{\beta}$ is subword of $\bar{\alpha}$, there are two subcases to consider.

Subcase 3.1. $q_{1}=\left.q_{2}\right|_{q}$ for some $q \in \mathfrak{M}(X)$. Then by Remark 3.5, $\left.q\right|_{\left\lfloor u_{1} u_{2}\right\rfloor}=\lfloor v\rfloor v$, i.e., $\left\lfloor u_{1} u_{2}\right\rfloor$ is a bracketed subword of $\lfloor v\rfloor v$. Note that $\lfloor v\rfloor v \neq\left\lfloor u_{1} u_{2}\right\rfloor$ by comparing the breadth. 
Subcase 3.1.1. $\left\lfloor u_{1} u_{2}\right\rfloor=\lfloor v\rfloor$. Then

$$
v=u_{1} u_{2},\left.q\right|_{\left\lfloor u_{1} u_{2}\right\rfloor}=\lfloor v\rfloor v=\left\lfloor u_{1} u_{2}\right\rfloor u_{1} u_{2} \text { and } q=\star u_{1} u_{2}
$$

and so

$$
\left.q_{1}\right|_{R(\alpha)}=\left.q_{1}\right|_{\left\lfloor u_{2}\right\rfloor\left\lfloor u_{1}\right\rfloor}=\left.q_{2}\right|_{\left.q\right|_{\left\lfloor u_{2}\right\rfloor\left\lfloor u_{1}\right\rfloor}}=\left.q_{2}\right|_{\left.\star u_{1} u_{2}\right|_{\left.\left\lfloor u_{2}\right\rfloor u_{1}\right\rfloor}}=\left.\left.\left.q_{2}\right|_{\left\lfloor u_{2}\right\rfloor\left\lfloor u_{1}\right\rfloor u_{1} u_{2}} \rightarrow_{\varphi} q_{2}\right|_{\left\lfloor u_{2}\right\rfloor u_{2}} \rightarrow_{\varphi} q_{2}\right|_{1} .
$$

Since $\left.q_{2}\right|_{R(\beta)}=\left.q_{2}\right|_{1}$, we get $\left.\left.q_{1}\right|_{R(\alpha)} \downarrow_{\Pi_{S}} q_{2}\right|_{R(\beta)}$.

Subcase 3.1.2. $\left\lfloor u_{1} u_{2}\right\rfloor$ is a bracketed subword of $v$, i.e., there exists $p \in \mathfrak{M}^{\star}(X)$ such that $v=$ $\left.p\right|_{\left\lfloor u_{1} u_{2}\right\rfloor}$. Then $q=\lfloor p\rfloor v$ or $q=\lfloor v\rfloor p$. If $q=\lfloor p\rfloor v$, then

$$
\left.q_{1}\right|_{R(\alpha)}=\left.q_{1}\right|_{\left\lfloor u_{2}\right\rfloor\left\lfloor u_{1}\right\rfloor}=\left.q_{2}\right|_{q_{\left\lfloor u_{2}\right\rfloor\left\lfloor u_{1}\right\rfloor}}=\left.q_{2}\right|_{\left\lfloor p\left\lfloor_{\left\lfloor u_{2}\right\rfloor\left\lfloor u_{1}\right\rfloor}\right\rfloor v\right.}=\left.\left.\left.q_{2}\right|_{\left\lfloor\left. p\left\lfloor_{\left\lfloor u_{2}\right\lrcorner\left\lfloor u_{1}\right\rfloor}\right\rfloor p\right|_{\left\lfloor u_{1} u_{2}\right\rfloor}\right.} \rightarrow_{\psi} q_{2}\right|_{\left.\left\lfloor\left. p\right|_{\left\lfloor u_{2}\right\lrcorner\left\lfloor u_{1}\right\rfloor}\right\rfloor p\right|_{\left.\left\lfloor u_{2}\right\rfloor u_{1}\right\rfloor}} \rightarrow_{\varphi} q_{2}\right|_{1},
$$

If $q=\lfloor v\rfloor p$, then

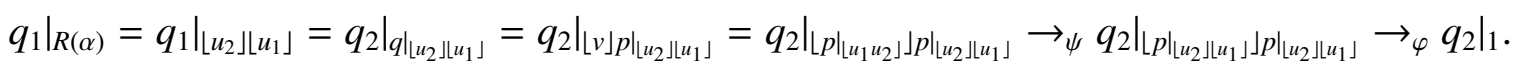

Since $\left.q_{2}\right|_{R(\beta)}=\left.q_{2}\right|_{1}$, we conclude that $\left.\left.q_{1}\right|_{R(\alpha)} \downarrow_{\Pi_{S}} q_{2}\right|_{R(\beta)}$.

Subcase 3.2. $q_{2}=\left.q_{1}\right|_{q}$ for some $q \in \mathfrak{M}^{\star}(X)$. Then by Remark 3.5, $\left.q\right|_{\lfloor v\rfloor v}=\left\lfloor u_{1} u_{2}\right\rfloor$, i.e., $\lfloor v\rfloor v$ is a bracketed subword of $\left\lfloor u_{1} u_{2}\right\rfloor$. Note that $\lfloor v\rfloor v \neq\left\lfloor u_{1} u_{2}\right\rfloor$. Thus we can assume $\lfloor v\rfloor v$ is a bracketed subword of $u_{1} u_{2}$.

Subcase 3.2.1. $\lfloor v\rfloor v$ is a bracketed subword of $u_{1}$ or $u_{2}$. By symmetry, we only need to consider the former. Then there exists $p \in \mathfrak{M}^{\star}(X)$ such that $u_{1}=\left.p\right|_{\lfloor v\rfloor v}$ and $q=\left\lfloor p u_{2}\right\rfloor$. Consequently,

$$
\begin{aligned}
& \left.q_{1}\right|_{R(\alpha)}=\left.q_{1}\right|_{\left\lfloor u_{2}\right\rfloor\left\lfloor u_{1}\right\rfloor}=\left.\left.q_{1}\right|_{\left\lfloor u_{2}\right\rfloor\left\lfloor\left. p\right|_{\lfloor v\rfloor v}\right\rfloor} \rightarrow{ }_{\varphi} q_{1}\right|_{\left\lfloor u_{2}\right\rfloor\left\lfloor\left. p\right|_{1}\right\rfloor}, \\
& \left.q_{2}\right|_{R(\beta)}=\left.q_{2}\right|_{1}=\left.q_{1}\right|_{\left.q\right|_{1}}=\left.\left.q_{1}\right|_{\left\lfloor\left. p\right|_{1} u_{2}\right\rfloor} \rightarrow_{\psi} q_{1}\right|_{\left\lfloor u_{2}\right\rfloor\left\lfloor\left. p\right|_{1}\right\rfloor},
\end{aligned}
$$

and so $\left.\left.q_{1}\right|_{R(\alpha)} \downarrow_{\Pi_{S}} q_{2}\right|_{R(\beta)}$.

Subcase 3.2.2. $\lfloor v\rfloor v$ is neither a bracketed subword of $u_{1}$ nor a bracketed subword of $u_{2}$. Then

$$
v=v_{1} v_{2}, u_{1}=u_{1}^{\prime}\lfloor v\rfloor v_{1} \text { and } u_{2}=v_{2} u_{2}^{\prime}
$$

for some $v_{1}, v_{2}, u_{1}^{\prime}, u_{2}^{\prime} \in \mathfrak{M}(X)$ with $v_{2} \neq 1$. Consequently,

$$
\left.\left\lfloor u_{1}^{\prime} \star u_{2}^{\prime}\right\rfloor\right\rfloor_{\lfloor v\rfloor v}=\left\lfloor u_{1}^{\prime}\lfloor v\rfloor v u_{2}^{\prime}\right\rfloor=\left\lfloor u_{1}^{\prime}\lfloor v\rfloor v_{1} v_{2} u_{2}^{\prime}\right\rfloor=\left\lfloor u_{1} u_{2}\right\rfloor=\left.q\right|_{\lfloor v\rfloor v} \text { and }\left\lfloor u_{1}^{\prime} \star u_{2}^{\prime}\right\rfloor=q \text {. }
$$

Thus

$$
\begin{aligned}
\left.q_{1}\right|_{R(\alpha)} & =\left.q_{1}\right|_{\left\lfloor u_{2}\right\rfloor\left\lfloor u_{1}\right\rfloor}=\left.\left.\left.q_{1}\right|_{\left\lfloor v_{2} u_{2}^{\prime}\right\rfloor\left\lfloor u_{1}^{\prime}\left\lfloor v_{1} v_{2}\right\rfloor v_{1}\right\rfloor} \rightarrow_{\psi} q_{1}\right|_{\left\lfloor v_{2} u_{2}^{\prime}\right\rfloor\left\lfloor u_{1}^{\prime}\left\lfloor v_{2}\right\rfloor\left\lfloor v_{1}\right\rfloor v_{1}\right\rfloor} \rightarrow_{\varphi} q_{1}\right|_{\left\lfloor v_{2} u_{2}^{\prime}\right\rfloor\left\lfloor u_{1}^{\prime}\left\lfloor v_{2}\right\rfloor\right\rfloor} \\
& \left.\left.\left.\left.\rightarrow_{\psi} q_{1}\right|_{\left\lfloor u_{2}^{\prime}\right\rfloor\left\llcorner v_{2}\right\rfloor\left\lfloor u_{1}^{\prime}\left\lfloor v_{2}\right\rfloor\right\rfloor} \rightarrow_{\psi} q_{1}\right|_{\left.\left\lfloor u_{2}^{\prime}\right\rfloor\left\lfloor v_{2}\right\rfloor\left\lfloor v_{2}\right\rfloor\right\rfloor\left\lfloor u_{1}^{\prime}\right\rfloor} \rightarrow_{\phi} q_{1}\right|_{\left.\left\lfloor u_{2}^{\prime}\right\rfloor v_{2}\right\rfloor v_{2}\left\lfloor u_{1}^{\prime}\right\rfloor} \rightarrow_{\varphi} q_{1}\right|_{\left\lfloor u_{2}^{\prime}\right\rfloor\left\lfloor u_{1}^{\prime}\right\rfloor,}, \\
\left.q_{2}\right|_{R(\beta)} & =\left.q_{2}\right|_{1}=\left.q_{1}\right|_{\left.q\right|_{1}}=\left.\left.q_{1}\right|_{\left\lfloor u_{1}^{\prime} u_{2}^{\prime}\right\rfloor} \rightarrow_{\psi} q_{1}\right|_{\left.\left\lfloor u_{2}^{\prime}\right\rfloor u_{1}^{\prime}\right\rfloor},
\end{aligned}
$$

and so $\left.\left.q_{1}\right|_{R(\alpha)} \downarrow_{\Pi_{S}} q_{2}\right|_{R(\beta)}$.

Case 4. $\bar{\alpha}=\psi\left(u_{1}, u_{2}\right)=\left\lfloor u_{1} u_{2}\right\rfloor$ and $\bar{\beta}=\chi(v)=v\lfloor v\rfloor$. This is similar to Case 3 .

Case 5. $\bar{\alpha}=\varphi(u)=\lfloor u\rfloor u$ and $\bar{\beta}=\varphi(v)=\lfloor v\rfloor v$. By symmetry, we may assume $q_{1}=\left.q_{2}\right|_{q}$ for some $q \in \mathfrak{M}^{\star}(X)$. From Remark 3.5, $\left.q\right|_{\lfloor u\rfloor u}=\lfloor v\rfloor v$, i.e., $\lfloor u\rfloor u$ is a bracketed subword of $\lfloor v\rfloor v$. If $\lfloor u\rfloor u=\lfloor v\rfloor v$, then $u=v, \alpha=\beta, q_{1}=q_{2}$ and so $\left.\left.q_{1}\right|_{R(\alpha)} \downarrow_{\Pi_{S}} q_{2}\right|_{R(\beta)}$. Suppose $\lfloor u\rfloor u \neq\lfloor v\rfloor v$. Since $\lfloor u\rfloor u \neq\lfloor v\rfloor,\lfloor u\rfloor u$ is a bracketed subword of $v$, i.e., there exists $p \in \mathfrak{M}^{\star}(X)$ such that $v=\left.p\right|_{\lfloor u\rfloor u}$. Then $q=\lfloor p\rfloor v$ or $q=\lfloor v\rfloor p$. If $q=\lfloor p\rfloor v$, then

$$
\left.q_{1}\right|_{R(\alpha)}=\left.q_{1}\right|_{1}=\left.q_{2}\right|_{\left.q\right|_{1}}=\left.q_{2}\right|_{\left\llcorner p \|_{1} v\right.}=\left.\left.\left.q_{2}\right|_{\left.\left\lfloor\left. p\right|_{1}\right\rfloor p\right|_{\lfloor u\rfloor u}} \rightarrow_{\varphi} q_{2}\right|_{\left.\left\llcorner\left. p\right|_{1}\right\rfloor p\right|_{1}} \rightarrow_{\varphi} q_{2}\right|_{1} .
$$

If $q=\lfloor p\rfloor v$, then

$$
\left.q_{1}\right|_{R(\alpha)}=\left.q_{1}\right|_{1}=\left.q_{2}\right|_{\left.q\right|_{1}}=\left.q_{2}\right|_{\left.\llcorner v\rfloor p\right|_{1}}=\left.\left.\left.q_{2}\right|_{\left.\left\lfloor\left. p\right|_{\lfloor u\rfloor u}\right\rfloor\right|_{1}} \rightarrow_{\varphi} q_{2}\right|_{\left.\left\llcorner\left. p\right|_{1}\right\rfloor p\right|_{1}} \rightarrow_{\varphi} q_{2}\right|_{1} .
$$

Since $\left.q_{2}\right|_{R(\beta)}=\left.q_{2}\right|_{1}$, we conclude that $\left.\left.q_{1}\right|_{R(\alpha)} \downarrow_{\Pi_{S}} q_{2}\right|_{R(\beta)}$. 
Case 6. $\bar{\alpha}=\chi(u)=u\lfloor u\rfloor$ and $\bar{\beta}=\chi(v)=v\lfloor v\rfloor$. This is similar to Case 5 .

Case 7. $\bar{\alpha}=\varphi(u)=\lfloor u\rfloor u$ and $\bar{\beta}=\chi(v)=v\lfloor v\rfloor$. This is also similar to Case 5 .

Theorem 3.15. Let $X$ be a set and $S$ the binary relation given in Eq. (6). With the monomial order given in [18], we have

(a) the term-rewriting system $\Pi_{S}$ is convergent.

(b) the set $\operatorname{Irr}(S)=\mathfrak{M}(X) \backslash \operatorname{Dom}\left(\Pi_{S}\right)$ is a section of the free group $\mathfrak{M}(X) /\langle S\rangle$.

Proof. (a) With a similar argument to the proof of Theorem $3.10(a)$, the result follows from Lemmas 3.12, 3.13 and 3.14.

(b) This part follows from Item (a) and Theorem 3.10 .

Remark 3.16. It is well known that reduced words are elements in the free group on a set $X$ [20]. The set $\operatorname{Irr}(S)=\mathfrak{M}(X) \backslash \operatorname{Dom}\left(\Pi_{S}\right)$ obtained in Theorem 3.15, of course, coincides with the set of reduced words.

Acknowledgements: The authors are supported by the National Natural Science Foundation of China (No. 11771191), the Fundamental Research Funds for the Central Universities (No. lzujbky2017-162), and the Natural Science Foundation of Gansu Province (Grant No. 17JR5RA175) and Shandong Province (No. ZR2016AM02).

We thank the anonymous referee for valuable suggestions helping to improve the paper.

\section{REFERENCES}

[1] M. Aguiar, On the associative analog of Lie bialgebras, J. Algebra 244 (2001), 492-532.

[2] F. Baader and T. Nipkow, 1998. Term Rewriting and All That, Cambridge U. P., Cambridge.

[3] C. Bai, A unified algebraic approach to the classical Yang-Baxter equations, J. Phys. A: Math. Theor. 40 (2007), 11073-11082.

[4] C. Bai, O. Bellier, L. Guo and X. Ni, Splitting of operations, Manin products and Rota-Baxter operators, Int. Math. Res. Not. IMRN. (2013), 485-524.

[5] G. Baxter, An analytic problem whose solution follows from a simple algebraic identity, Pacific J. Math. 10 (1960), 731-742.

[6] L. A. Bokut and Y. Chen, Gröbner-Shirshov bases and their calculation, Bull. Math. Sci. 4 (2014), 325395.

[7] L. A. Bokut, Y. Chen and J. Qiu, Gröbner-Shirshov bases for associative algebras with multiple operators and free Rota-Baxter algebras, J. Pure Appl. Algebra 214 (2010), 89-110.

[8] J. Cariñena, J. Grabowski and G. Marmo, Quantum bi-Hamiltonian systems, Internat. J. Modern Phys. A 15 (2000), 4797-4810.

[9] A. Connes and D. Kreimer, Renormalization in quantum field theory and the Riemann-Hilbert problem. I. The Hopf algebra structure of graphs and the main theorem, Comm. Math. Phys. 210 (2000), 249-273.

[10] P. M. Cohn, Further algebra and applications, Springer-Verlag London Ltd., London, 2003.

[11] A. Connes and D. Kreimer, Renormalization in quantum field theory and the Riemann-Hilbert problem. I. The Hopf algebra structure of graphs and the main theorem, Comm. Math. Phys. 210 (2000), 249-273.

[12] K. Ebrahimi-Fard, L. Guo and D. Kreimer, Spitzer's identity and the algebraic Birkhoff decomposition in pQFT, J. Phys. A: Math. Gen. 37 (2004), 11037-11052.

[13] X. Gao and L. Guo, Rota's Classification Problem, rewriting systems and Gröbner-Shirshov bases, J. Algebra 470 (2017), 219-253.

[14] X. Gao, L. Guo, W. Sit and S. Zheng, Rota-Baxter type operators, rewriting systems and GröbnerShirshov bases, J. Symbolic Computation, to appear, arXiv:1412.8055v1.

[15] X. Gao and T. Zhang, Averaging algebras, rewriting systems and Gröbner-Shirshov bases, Journal of Algebra and Its Applications 16(2) (2018), 1850130.

[16] L. Guo, Operated semigroups, Motzkin paths and rooted trees, J. Algebraic Combinatorics 29 (2009), 35-62. 
[17] L. Guo, An Introduction to Rota-Baxter Algebra, International Press (US) and Higher Education Press (China), 2012.

[18] L. Guo, W. Sit and R. Zhang, Differential Type Operators and Gröbner-Shirshov Bases, J. Symb. Comput. 52 (2013), 97-123.

[19] L. Guo and B. Zhang, Renormalization of multiple zeta values, J. Algebra 319 (2008), 3770-3809.

[20] T. W. Hungerford, Algebras, Springer, 1974.

[21] J. M. Howie, Fundamentals of Semigroup Theory, Clarendon Press, Oxford, 1995.

[22] E. Kolchin, Differential algebraic groups, Academic Press, Inc., Orlando, FL, 1985.

[23] A. G. Kurosh, Free sums of multiple operator algebras, Siberian. Math. J. 1 (1960), $62-70$ (in Russian).

[24] M. V. Lawson, Inverse Semigroups: The Theory of Partial Symmetries, World Scientific, 1998.

[25] P. Leroux, On some remarkable operads constructed from Baxter operators, preprint, Nov. 2003, arXiv:math.QA/0311214.

[26] J. B. Miller, Averaging and Reynolds operators on Banach algebra I, Representation by derivation and antiderivations, J. Math. Anal. Appl. 14 (1966), 527-548.

[27] E. Ohlebusch, Advanced topics in term rewriting, Springer, New York, 2002.

[28] J. F. Ritt, Differential Algebra, American Mathematical Society, New York, 1950.

[29] S. Zheng and L. Guo, Relative locations of subwords in free operated semigroups and Motzkin words, Frontier Math. 10 (2015), 1243-1261.

School of Mathematics and Statistics, Lanzhou University, Lanzhou, 730000, P.R. China

E-mail address: zj_10@1zu.edu.cn

School of Mathematics and Statistics, Key Laboratory of Applied Mathematics and Complex Systems, Lanzhou University, Lanzhou, 730000, P.R. China

E-mail address: gaoxing@lzu.edu.cn 